\title{
THE STRUCTURE AND LIMITING BEHAVIOR OF LOCALLY OPTIMAL MINIMIZERS
}

\author{
Moshe MARCUS, Alexander J. ZASLAVSKI
}

Department of Mathematics, Technion, Haifa 32000, Israel

Received 12 January 2001

RÉSUMÉ. - Nous étudions la structure des minimiseurs localement optimaux ( -øptimaux) d'une classe de problèmes variationelles du second ordre sur $R_{+}$. Ces problèmes sont liés à un modèle de thermodinamique introduit dans [4]. Nous montrons que si $v$ est um minimiseur non-périodique $c$-optimal, alors la courbe correspondante dans l'espace des phases ne s'autointersecte pas. En utilisant ce fait, nous étudions le comportement asymptotique à l'infini des minimiseurs $c$-optimaux, et la structure de leurs ensembles limites.

(C) 2002 L'Association Publications de l'Institut Henri Poincaré. Published by Elsevier B.V. All rights reserved

ABSTRACT. - We study the structure of locally optimal (c-optimal) minimizers of a class of second order variational problems on $R_{+}$. The problems are related to a model in thermodynamics introduced in [4]. We show that if $v$ is a $c$-optimal nonperiodic minimizer, then the corresponding curve in the phase plane does not intersect itself. Using this fact we study the asymptotic behavior at infinity of $c$-optimal minimizers, and the structure of their limiting sets. (ㅇ) 2002 L'Association Publications de l'Institut Henri Poincaré. Published by Elsevier B.V. All rights reserved

Math. Subj. Class. (1991): 49J99; 58F99

Keywords: Infinite horizon problems; $c$-optimal minimizers; Limiting set

\section{Introduction}

In this paper we investigate the structure of locally optimal solutions of infinite horizon variational problems associated with the functional

$$
I^{f}(D ; w)=\int_{D} f\left(w(t), w^{\prime}(t), w^{\prime \prime}(t)\right) \mathrm{d} t, \quad \forall w \in W^{2,1}(D),
$$

where $D$ is a bounded interval on the real line and $f \in C\left(R^{3}\right)$ belongs to a space of functions $\mathfrak{M}$ to be described below.

We shall consider the problems

$$
\inf \left\{I^{f}(D ; w): w \in W^{2,1}(D),\left(w, w^{\prime}\right)\left(T_{1}\right)=x,\left(w, w^{\prime}\right)\left(T_{2}\right)=y\right\} \quad\left(P_{D}^{x, y}\right)
$$


for $D=\left(T_{1}, T_{2}\right)$ and $x, y \in R^{2}$. We shall also consider the following problem on the half line:

$$
\inf \left\{J^{f}(w): w \in W_{l o c}^{2,1}(0, \infty), f\left(w, w^{\prime}, w^{\prime \prime}\right) \in L^{1}(0, T), \forall T>0\right\}
$$

where

$$
J^{f}(w)=\liminf _{T \rightarrow \infty} T^{-1} I^{f}((0, T) ; w) .
$$

Variational problems of this type were considered in [6,9,12-14]. Similar constrained problems (involving a mass constraint), were studied in $[4,7,8,10]$. The constrained problems were conceived as models for determining the thermodynamical equilibrium states of unidimensional bodies involving 'second order' materials for which the free energy density is given by $f$. A discussion of the physics underlying these models can be found in Coleman [2,3] and in Coleman, Marcus and Mizel [4] which initiated a systematic study of the corresponding constrained variational problem on $R$. Properties of minimizers of the mass constrained problem on bounded intervals, and their relation to minimizers of the limiting problem on the full line were studied by Marcus $[7,8]$ and Marcus and Zaslavski [10].

In the present paper we study the unconstrained problem $\left(P_{\infty}\right)$ and related problems on bounded intervals. One of our main goals is to describe the limiting set of $c$-optimal minimizers of $\left(P_{\infty}\right)$, (see definition below). In the remaining part of this introduction we discuss various results concerning the unconstrained problem. But first we describe the space of integrands $\mathfrak{M}$ that we are going to consider.

Let $a=\left(a_{1}, a_{2}, a_{3}, a_{4}\right) \in R^{4}, a_{i}>0, i=1,2,3,4$ and let $\alpha, \beta, \gamma$ be real numbers such that $1 \leqslant \beta<\alpha, \beta \leqslant \gamma$ and $\gamma>1$. Denote by $\mathfrak{M}=\mathfrak{M}(\alpha, \beta, \gamma, a)$ the family of continuous functions $\{f\}$ such that

$$
\begin{aligned}
& \text { (i) } f \in C^{2}\left(R^{3}\right), \partial f / \partial x_{2} \in C^{2}\left(R^{3}\right), \partial f / \partial x_{3} \in C^{3}\left(R^{3}\right), \\
& \text { (ii) } \partial^{2} f / \partial x_{3}^{2}>0, \\
& \text { (iii) } f(x) \geqslant a_{1}\left|x_{1}\right|^{\alpha}-a_{2}\left|x_{2}\right|^{\beta}+a_{3}\left|x_{3}\right|^{\gamma}-a_{4}, \\
& \text { (iv) }(|f|+|\nabla f|)(x) \leqslant M_{f}\left(\left|x_{1}\right|+\left|x_{2}\right|\right)\left(1+\left|x_{3}\right|^{\gamma}\right), \quad \forall x \in R^{3},
\end{aligned}
$$

where $M_{f}:[0, \infty) \mapsto[0, \infty)$ is a continuous function depending on $f$.

In the sequel we assume that $f \in \mathfrak{M}=\mathfrak{M}(\alpha, \beta, \gamma, a)$ where $(\alpha, \beta, \gamma, a)$ is an arbitrary but fixed set of parameters satisfying the above conditions. Conditions (1.1)(iii), (iv) imply that,

$$
w \in W_{l o c}^{2,1}\left(R_{+}\right) \quad \text { and } \quad f\left(w, w^{\prime}, w^{\prime \prime}\right) \in L^{1}(0, T), \quad \forall T>0 \Leftrightarrow w \in W_{l o c}^{2, \gamma}[0, \infty),
$$

where

$$
W_{\text {loc }}^{2, \gamma}[0, \infty)=\left\{w \in W_{\text {loc }}^{2, \gamma}(0, \infty): w \in W^{2, \gamma}(0, T), \forall T>0\right\} .
$$

For every $f \in \mathfrak{M}$, the infimum in $\left(P_{\infty}\right)$ is finite (see [6] or [9, Lemma 2.2]). Put

$$
\mu(f):=\inf \left(P_{\infty}\right) .
$$


Leizarowitz and Mizel [6] showed that, if $f$ satisfies the condition

$$
\mu(f)<\inf _{(w, s) \in R^{2}} f(w, 0, s),
$$

then $\left(P_{\infty}\right)$ possesses a periodic minimizer. Later, Zaslavski [12] proved that this conditon is not needed: the result holds for all $f \in \mathfrak{M}$.

For $w \in W^{2, \gamma}(D), D$ a bounded interval, put

$$
E^{f}(D, w):=I^{f}(D, w)-\mu(f)|D| .
$$

By definition, $w \in W_{l o c}^{2, \gamma}[0, \infty)$ is a minimizer of $\left(P_{\infty}\right)$ iff $\liminf _{T \rightarrow \infty} \frac{1}{T} E^{f}((0, T), w)=$ 0 . If, in addition, $\left\{E^{f}((0, T), w): T>0\right\}$ is bounded we say that $w$ is an $f$-good minimizer. This concept was first introduced by Leizarowitz [5] in a discrete context. More generally, if $v \in W_{l o c}^{2, \gamma}(U)$ for some unbounded interval $U$, and if there exists a constant $M=M(U, v)$ such that $\left|E^{f}(D, v)\right| \leqslant M$ for every bounded interval $D \subset U$, we say that $v$ is an $f$-good function in $U$. The family of $f$ good functions in $U$ is denoted by $\mathcal{G}^{f}(U)$; the family of $f$-good minimizers (i.e. $\mathcal{G}^{f}\left(R_{+}\right)$) will be denoted briefly by $\mathcal{G}^{f}$.

The following result was obtained in [12, Theorem 3.1]; a discrete version was previously established in [5].

For every $w \in W_{\text {loc }}^{2, \gamma}[0, \infty)$, either $\left\{\left|E^{f}((0, T), w)\right|: T>0\right\}$ is bounded, i.e., $w \in \mathcal{G}^{f}$, or $\lim _{T \rightarrow \infty} E^{f}((0, T), w)=\infty$. If $w \in \mathcal{G}^{f}$ then $w \in W^{1, \infty}\left(R_{+}\right)$.

If $w \in W_{l o c}^{2, \gamma}(U) \cap W^{1, \infty}(U)$, where $U$ is an unbounded interval, we say that $v$ is $c$ optimal on $U$, if, for every bounded interval $D=\left(T_{1}, T_{2}\right) \subset U$, the restriction $\left.w\right|_{D}$ is a minimizer of $\left(P_{D}^{x, y}\right)$ with $x=\left(w, w^{\prime}\right)\left(T_{1}\right), y=\left(w, w^{\prime}\right)\left(T_{2}\right)$. The family of $c$-optimal functions on $U$ is denoted by $\mathcal{T}^{f}(U)$; the family of $c$-optimal functions on $R_{+}$is denoted briefly by $T^{f}$.

Note that the definition of a $c$-optimal function does not assume that it is a minimizer of $\left(P_{\infty}\right)$. However, by [9, Proposition 2.3]:

If $w$ is c-optimal on $R_{+}$then it is an $(f)$-good minimizer.

Clearly, if $u \in \mathcal{T}^{f}$, then $u$ satisfies the Euler-Lagrange equation associated with the functional $I^{f}$, namely,

$$
\frac{\partial f}{\partial x_{1}}\left(u, u^{\prime}, u^{\prime \prime}\right)-\frac{\mathrm{d}}{\mathrm{d} t}\left(\frac{\partial f}{\partial x_{2}}\left(u, u^{\prime}, u^{\prime \prime}\right)\right)+\frac{\mathrm{d}^{2}}{\mathrm{~d} t^{2}}\left(\frac{\partial f}{\partial x_{3}}\left(u, u^{\prime}, u^{\prime \prime}\right)\right)=0 .
$$

This is a fourth order, autonomous, quasi linear equation in $u$ whose main coefficient is $\frac{\partial^{2} f}{\partial x_{3}^{2}}\left(u, u^{\prime}, u^{\prime \prime}\right)$. By assumption (1.1)(ii), this coefficient is positive everywhere. Consequently, if $u_{1}, u_{2}$ are $c$-optimal minimizers on $R_{+}$such that, at some point $t_{0}$, $u_{1}^{(j)}\left(t_{0}\right)=u_{2}^{(j)}\left(t_{0}\right)$ for $j=0,1,2,3$ then $u_{1} \equiv u_{2}$.

The class of $c$-optimal minimizers $\mathcal{T}^{f}$ is, in a sense, a 'small' subset of $\mathcal{G}^{f}$. Obviously, a $c$-optimal minimizer cannot be modified on compact sets without losing the property of $c$-optimality. On the other hand the property of $f$-goodness is stable with respect to 
such modifications. Indeed, if $w_{0} \in \mathcal{G}^{f}$ and if $w_{1}$ is a function in $W_{l o c}^{2, \gamma}[0, \infty)$ such that $\left\{x \in R_{+}: w_{0}(x) \neq w_{1}(x)\right\}$ is bounded, then $w_{1} \in \mathcal{G}^{f}$.

Nevertheless the class of $c$-optimal minimizers on $R_{+}$is a 'large' class in the following sense:

Proposition 1.1. - For every point $x=\left(x_{1}, x_{2}\right) \in R^{2}$ there exists a c-optimal minimizer $w$ on $R_{+}$such that $\left(w(0), w^{\prime}(0)\right)=x$.

Such a minimizer can be constructed as follows. Let $w_{T}$ be a minimizer of the problem

$$
\inf \left\{I^{f}(D ; w): w \in W^{2,1}(D),\left(w, w^{\prime}\right)(0)=x\right\},
$$

for $D=(0, T)$. By [10, Corollary 3.3] and [9, Lemma 2.2], there exists a positive constant $M$ such that

$$
\left\|w_{T}\right\|_{W^{2, \gamma}(s, s+1)} \leqslant M, \quad \forall T>0, \forall s \in(0, T-1) .
$$

Therefore, if $T_{n} \rightarrow \infty$, then $\left\{w_{T_{n}}\right\}$ possesses a subsequence which converges in $C^{1}(D)$ and converges weakly in $W^{2, \gamma}(D)$, for every bounded interval $D \subset R_{+}$. By a standard lower semicontinuity argument (see e.g. the proof of Lemma 2.3 in [9]) the limiting function $w$ is $c$-optimal on $R_{+}$and $\left(w(0), w^{\prime}(0)\right)=x$.

It is interesting to note that, in general, a $c$-optimal function on $R_{+}$cannot be extended to a $c$-optimal function on $R$. In this sense, the class of $c$-optimal functions on $R$, which we denote by $\mathcal{T}^{f}(R)$, is much more restricted than $\mathcal{T}^{f}$. In fact, $\mathcal{T}^{f}(R)$ is a bounded set in $W^{1, \infty}(R)$ (see Lemma 3.7 below) while, by our previous assertion, $\mathcal{T}^{f}$ is unbounded in $W^{1, \infty}\left(R_{+}\right)$. In a generic sense the contrast is even more striking: $\mathcal{T}^{f}(R)$ is precisely the set of translates of a single periodic minimizer. Indeed, there exists a dense subset of $\mathfrak{M}$ such that, for each $f$ in this subset, problem $\left(P_{\infty}\right)$ possesses a unique (up to translation) periodic minimizer and satisfies the asymptotic turnpike property or (ATP) (see [9, Theorems 3.1, 3.2]). Further, by [9, Theorem 2.1], (ATP) implies the strong turnpike property or (STP) (see [9, Definition 1.2]). Finally, (STP) implies, in a straightforward manner, that every $c$-optimal function on $R$ is a translate of the (unique) periodic minimizer.

Another class of minimizers, which plays an important role in our theory, is the class of perfect minimizers, which is a subclass of $\mathcal{T}^{f}$. First we define the concept of a perfect function on an arbitrary interval. The definition requires some additional notation. For every $w \in \mathcal{G}^{f}$, put

$$
E_{\infty}^{f}(w):=\liminf _{T \rightarrow \infty} E^{f}((0, T), w) .
$$

In a sense, $E_{\infty}^{f}(w)$ measures the distance between $I^{f}((0, T), w)$ and the target value $T \mu(f)$ as $T \rightarrow \infty$. For every $x \in R^{2}$, put

$$
\pi^{f}(x):=\inf \left\{E_{\infty}^{f}(w): w \in \mathcal{G}^{f},\left(w(0), w^{\prime}(0)\right)=x\right\} .
$$

It is known that $\pi^{f} \in C\left(R^{2}\right)$ and $\pi^{f}(x) \rightarrow \infty$ as $|x| \rightarrow \infty$, [6]. If $v \in W^{2, \gamma}(D)$, $D=\left(T_{1}, T_{2}\right)$, put

$$
\Gamma^{f}(D, v):=I^{f}(D ; v)-|D| \mu(f)+\pi^{f}\left(X_{v}\left(T_{2}\right)\right)-\pi^{f}\left(X_{v}\left(T_{1}\right)\right) .
$$


If $\left\{D_{j}\right\}_{j=1}^{k}$ is a partition of $D$ into disjoint subintervals, then, by (1.4),

$$
\Gamma^{f}(D, v)=\sum_{j=1}^{k} \Gamma^{f}\left(D_{j}, v\right)
$$

We refer to this property of $\Gamma$ as additivity on intervals.

Given $x, y \in R^{2}$ and $T>0$, let $U_{T}^{f}(x, y)$ denote the infimum in problem $\left(P_{(0, T)}^{x, y}\right)$. Then

$$
\Gamma^{f}((0, T), v) \geqslant U_{T}^{f}(x, y)-T \mu(f)+\pi(y)-\pi(x)=: \Theta_{T}^{f}(x, y),
$$

for every $v \in W^{2, \gamma}(0, T)$ such that $\left(v(0), v^{\prime}(0)\right)=x$ and $\left(v(T), v^{\prime}(T)\right)=y$. The following result, obtained by Leizarowitz and Mizel [6, Section 4], adapts to the present problem a general principle concerning cost functions in infinite horizon problems, due to Leizarowitz [5, Proposition 5.1].

$\Theta_{T}^{f}$ is non-negative and, for every $T>0$ and every $x \in R^{2}$, there exists $y \in R^{2}$ such that $\Theta_{T}^{f}(x, y)=0$.

If $D$ is a bounded interval and $w \in W^{2, \gamma}(D)$, then $w$ is $f$-perfect on $D$ if $\Gamma^{f}(D, w)=$ 0 . If $U$ is an unbounded interval, we say that $w$ is $f$-perfect on $U$ if $w$ is $f$-perfect on $D$ for every bounded interval $D \subset U$. The family of $f$-perfect functions on $U$ is denoted by $\mathcal{P}^{f}(U)$; the family of $f$-perfect functions on $R_{+}$is denoted briefly by $\mathcal{P}^{f}$.

If $w$ is $f$-perfect on $D=\left(T_{1}, T_{2}\right)$ then: (a) $w$ is a minimizer of problem $\left(P_{D}^{x, y}\right)$ where $x=\left(w, w^{\prime}\right)\left(T_{1}\right), y=\left(w, w^{\prime}\right)\left(T_{2}\right)$ and (b) $w$ is perfect on every subinterval of $D$. These assertions follow immediately from the non-negativity of $\Theta_{T}^{f}$ and the additivity of $\Gamma^{f}$. Note also that the result of [6] quoted above, implies the following.

Proposition 1.2. - For every $x \in R^{2}$ there exists a perfect function $v$ on $R_{+}$such that $\left(v(0), v^{\prime}(0)\right)=x$.

The function $v$ can be constructed inductively on the intervals $(0, n), n=1,2, \ldots$, as follows. Let $y \in R^{2}$ be a point such that $\Theta_{1}(x, y)=0$ and let $\left.v\right|_{(0,1)}$ be a minimizer of $\left(P_{(0,1)}^{x, y}\right)$. Now suppose that $v$ was defined as a perfect function on the interval $(0, n)$. Put $z=\left(v, v^{\prime}\right)(n)$. and let $\zeta \in R^{2}$ be such that $\Theta_{1}(z, \zeta)=0$. Finally define $v$ on $(n, n+1)$ so that it is a minimizer of $\left(P_{(n, n+1)}^{z, \zeta}\right)$. The additivity property of $\Gamma^{f}$ guarantees that the function $v$ constructed in this way is $f$-perfect on every interval $(0, n), n=1,2, \ldots$, and consequently on $R_{+}$.

The definition of a perfect function does not require boundedness. However the following result holds:

PROPOSITION 1.3. - (i) If $w$ is $f$-perfect on $R_{+}$, then $w \in W^{1, \infty}\left(R_{+}\right)$.

(ii) Every $f$-perfect function on $R_{+}$is a c-optimal minimizer of $\left(P_{\infty}\right)$.

Indeed, by assumption, $E^{f}((0, T), w)=\pi^{f}\left(\left(w, w^{\prime}\right)(0)\right)-\pi^{f}\left(\left(w, w^{\prime}\right)(T)\right)$, for every $T>0$. By [12, Theorem 3.1], $\inf _{T>0} E^{f}((0, T), w)>-\infty$. Hence $\sup _{T>0} \pi^{f}\left(X_{w}(T)\right)<$ $\infty$. Since it is known that $\pi^{f}(x) \rightarrow \infty$ as $|x| \rightarrow \infty$, this fact implies assertion (i). The second assertion is an immediate consequence of (i) and the definition of perfect functions. 
Obviously, every periodic minimizer of $\left(P_{\infty}\right)$ is $f$-perfect. Let $\mathcal{S}^{f}$ denote the class of periodic minimizers. Then

$$
\mathcal{S}^{f} \subset \mathcal{P}^{f} \subset \mathcal{T}^{f} \subset \mathcal{G}^{f} .
$$

We note that, for every $f, \mathcal{S}^{f}$ is a proper subset of $\mathcal{P}^{f}$. Indeed, by [9, Proposition 2.3], $\mathcal{S}^{f}$ is bounded in $W^{1, \infty}\left(R_{+}\right)$. On the other hand, by Proposition 1.2, $\mathcal{P}^{f}$ is unbounded in the norm of $W^{1, \infty}\left(R_{+}\right)$. Obviously, $\mathcal{T}^{f}$ is a proper subset of $\mathcal{G}^{f}$. An interesting question is wether there exist $c$-optimal minimizers which are not perfect. The answer depends on the integrand $f$. If $f$ possesses the periodic uniqueness property, i.e. $\left(P_{\infty}\right)$ has a unique (up to translation) periodic minimizer, then, by Theorem 5.1 below, $\mathcal{P}^{f}=\mathcal{T}^{f}$. However, there exists a family of integrands f for which $\mathcal{P}^{f}$ is a proper subset of $\mathcal{T}^{f}$. A construction of such a family of integrands and other results pertaining to the nonuniqueness case will be presented in a subsequent paper.

We turn now to a description of the main results of the present paper. The first main result concerns the non-intersecting property of $c$-optimal minimizers.

THEOREM 1.1. - (a) Let $v$ be a c-optimal minimizer of $\left(P_{\infty}\right)$. If there exists $T>0$ such that

$$
\left(v, v^{\prime}\right)(0)=\left(v, v^{\prime}\right)(T)
$$

then $v$ is periodic with period $T$.

(b) Let $v_{1}, v_{2}$ be c-optimal minimizers of $\left(P_{\infty}\right)$ such that

$$
\left(v_{1}, v_{1}^{\prime}\right)(0)=\left(v_{2}, v_{2}^{\prime}\right)(0)
$$

If there exist $t_{1}, t_{2} \in[0, \infty)$ such that $\left(t_{1}, t_{2}\right) \neq(0,0)$ and

$$
\left(v_{1}, v_{1}^{\prime}\right)\left(t_{1}\right)=\left(v_{2}, v_{2}^{\prime}\right)\left(t_{2}\right),
$$

then $v_{1} \equiv v_{2}$.

Remark. - In the case of perfect minimizers the non-intersecting property is known. Part (a) is implicitly contained in [6, Proposition 5.3]; for the full result see [9, Lemma 2.8]. In this case the the non-intersecting property is a simple consequence of the uniqueness of solutions of the initial value problem for (EL). In the case of $c$-optimal minimizers, the non-intersecting property goes much deeper. One of the ingredients in its proof is the following interesting property of $c$-optimal minimizers (see Proposition 4.2 below):

Let $v$ be a c-optimal minimizer. If $T>0, x=\left(v, v^{\prime}\right)(0), y=\left(v, v^{\prime}\right)(T)$, then

$$
\Theta_{T}(x, y)=\inf _{S>0} \Theta_{S}(x, y) .
$$

The next two results describe the limiting set of $c$-optimal minimizers in the phase plane and their asymptotic behaviour at infinity. The non-intersecting property plays a crucial role in the derivation of these results. We use the following notation. If $v \in W_{l o c}^{2,1}[0, \infty) \cap W^{1, \infty}(0, \infty)$, then the set of limiting points of $\left(v, v^{\prime}\right)$ as $t \rightarrow \infty$ is denoted by $\Omega(v)$. 
THEOREM 1.2. - Let

$$
\mu(f)<\inf \left\{f(x, 0,0): x \in R^{1}\right\}
$$

and let $v$ be a c-optimal minimizer of $\left(P_{\infty}\right)$. Then there exists a periodic minimizer $w$ of $\left(P_{\infty}\right)$ such that $\Omega(v)=\Omega(w)$ and the following assertion holds:

Let $T>0$ be a period of $w$. Then, for every $\varepsilon>0$ there exists $\tau(\varepsilon)>0$ such that for every $\tau \geqslant \tau(\varepsilon)$ there exists $s \in[0, T)$ such that,

$$
\left|\left(v, v^{\prime}\right)(t+\tau)-\left(w, w^{\prime}\right)(s+t)\right| \leqslant \varepsilon, \quad t \in[0, T] .
$$

Remark. - In the case that problem $\left(P_{\infty}\right)$ possesses a unique (up to translation) periodic minimizer, this result was proved in [9].

In the case that $v$ is a perfect minimizer, it was shown in [6] that the limiting set $\Omega(v)$ contains $\Omega(w)$ for some periodic minimizer $w$. Combining this fact with the nonintersecting property for perfect minimizers, it is not difficult to establish the theorem in this case.

In the special case of the integrand $f\left(u, u^{\prime}, u^{\prime \prime}\right)=\left(u^{\prime \prime}\right)^{2}-b\left(u^{\prime}\right)^{2}+\left(u^{2}-1\right)^{2}(b$ a positive constant), an assertion referring to the limiting behaviour at $\pm \infty$ of 'minimal energy configurations' (i.e., $c$-optimal functions) on the whole line appears in the introduction to [11]. The nature of the limiting process was not specified and proof was not supplied. The techniques used in [11] for the proof of other results depended on the symmetries associated with this specific integrand.

Our next result describes the structure of the limiting set of $c$-optimal minimizers, in the absence of assumption (N). In this case the structure of the limiting set is considerably more complicated. This result is new even in the case of perfect functions.

THEOREM 1.3. - Suppose that $\mu(f)=\inf \left\{f(d, 0,0): d \in R^{1}\right\}$ and that the set $\mathfrak{D}=$ $\left\{d \in R^{1}: f(d, 0,0)=\mu(f)\right\}$ is finite. Let $v$ be a c-optimal minimizer of $\left(P_{\infty}\right)$. Then $\Omega(v)$ is a compact connected set and the following alternative holds. Either there exists a periodic minimizer $w$ such that $\Omega(v)=\Omega(w)$ and (1.8) holds, or $\Omega(v)$ is a finite union of arcs $\bigcup_{j=1}^{k} \bar{\Xi}_{j}$ such that each arc $\Xi_{j}$ is the phase plane image of a perfect function $u_{j}$, i.e.,

$$
\Xi_{j}=\left\{\left(u_{j}, u_{j}^{\prime}\right)(t): t \in R^{1}\right\}, \quad j=1, \ldots, k .
$$

Furthermore, each function $u_{j}$ is monotone in neighborhoods of $+\infty$ and $-\infty$ and satisfies,

$$
\lim _{t \rightarrow \infty}\left(u_{j}, u_{j}^{\prime}\right)(t) \in \mathfrak{D} \times\{0\}, \quad \lim _{t \rightarrow-\infty}\left(u_{j}, u_{j}^{\prime}\right)(t) \in \mathfrak{D} \times\{0\}
$$

\section{Preliminaries}

For every $T>0$ and $x, y \in R^{2}$ put

$U_{T}^{f}(x, y)=\inf \left\{I^{f}(0, T, w): w \in W^{2, \gamma}(0, T),\left(w, w^{\prime}\right)(0)=x,\left(w, w^{\prime}\right)(T)=y\right\}$. 
For $x \in R^{n}, B \subset R^{n}$ put $d(x, B):=\inf \{|x-y|: y \in B\}$ (where $|\cdot|$ is the Euclidean norm) and denote by $\operatorname{dist}(A, B)$ the distance in the Hausdorff metric between two subsets $A, B$ of $R^{n}$. If $v \in W^{2,1}(D)$ put,

$$
X_{v}(t)=\left(v(t), v^{\prime}(t)\right), \quad t \in D .
$$

Since in the present paper we consider an arbitrary but fixed function $f \in \mathfrak{M}$ the superscript $f$ will be ommitted in notation such as $I^{f}, \Gamma^{f}$ etc.

The following result, derived in [6, Section 4], is based on a general principle concerning cost functions in infinite horizon problems, established in [5, Proposition 5.1].

Proposition 2.1. - Let $\pi^{f}$ be defined as in (1.3) and $U_{T}^{f}$ as in (2.1). Then $\pi^{f} \in$ $C\left(R^{2}\right)$ and $(T, x, y) \rightarrow U_{T}^{f}(x, y)$ is continuous in $(0, \infty) \times R^{2} \times R^{2}$. Furthermore, for every $T, x, y$ as above,

$$
\Theta_{T}^{f}(x, y)=U_{T}^{f}(x, y)-T \mu(f)-\left(\pi^{f}(x)-\pi^{f}(y)\right) \geqslant 0,
$$

and, for every $T>0$ and every $x \in R^{2}$, there exists $y \in R^{2}$ such that $\Theta_{T}^{f}(x, y)=0$.

The following simple but useful result was established in [9]. The brief proof is repeated below.

Proposition 2.2. - Let $D=\left(T_{1}, T_{2}\right)$ be a bounded interval and suppose that $w_{1}, w_{2}$ are perfect functions in $D$. If there exists $\tau \in D$ such that $\left(w_{1}, w_{1}^{\prime}\right)(\tau)=\left(w_{2}, w_{2}^{\prime}\right)(\tau)$ then $w_{1}=w_{2}$ everywhere in $D$.

Proof. - We define a function $u$ in $D$ as follows:

$$
u(t)=w_{1}(t), \quad t \in\left[T_{1}, \tau\right], \quad u(t)=w_{2}(t), \quad t \in\left(\tau, T_{2}\right] .
$$

Evidently $u \in W^{2,1}(D)$ and $\Gamma(D ; u)=\Gamma\left(\left(T_{1}, \tau\right), u\right)+\Gamma\left(\left(\tau, T_{2}\right), u\right)=0$. Consequently $u$ is a minimizer of problem $\left(P_{D}^{x, y}\right)$ with $x=\left(u, u^{\prime}\right)\left(T_{1}\right)$ and $y=\left(u, u^{\prime}\right)\left(T_{2}\right)$. Since $u$, $w_{1}, w_{2}$ satisfy the Euler-Lagrange equation (EL) and $u$ coincides with $w_{1}$ on $\left(T_{1}, \tau\right)$ and with $w_{2}$ on $\left(\tau, T_{2}\right)$, we conclude that $u=w_{1}=w_{2}$ everywhere in $D$.

The next result is of a more technical nature but it plays a central role in our arguments.

PROPOSITION 2.3. - Let $v \in W_{\text {loc }}^{2,1}[0, \infty)$ be a good function. Let $\left\{\xi_{k}\right\}$ be a sequence in $(0, \infty)$ such that $\xi_{k} \rightarrow \infty$ and let $u_{k}, k=1,2, \ldots$ be the function given by

$$
u_{k}(t)=v\left(t+\xi_{k}\right) \quad\left(-\xi_{k} \leqslant t<\infty\right) .
$$

Then there exists a subsequence $\left\{u_{k_{j}}\right\}$ and a function $u \in W_{l o c}^{2, \gamma}\left(R^{1}\right)$ such that

$$
\begin{aligned}
& \text { (a) } u_{k_{j}} \rightarrow u \text { weakly in } W^{2, \gamma}(-T, T), \forall T>0, \\
& \text { (b) }\left\{\left(u, u^{\prime}\right)(t): t \in R^{1}\right\} \subset \Omega(v), \\
& \text { (c) } \Gamma\left(\left(T_{1}, T_{2}\right) ; u\right)=0, \quad \forall T_{1}, T_{2} \in R^{1} .
\end{aligned}
$$


Thus $u$ is a perfect function on $R^{1}$. If, in addition, there exists $\tau \in R^{1}$ such that the sequence $\left\{X_{v}\left(\tau+\xi_{k}\right)\right\}$ converges, then

$$
u_{k} \rightarrow u \quad \text { weakly in } W^{2, \gamma}(-T, T), \forall T>0 .
$$

Proof. - For every $T>0$ choose an integer $k_{T}$ such that $T<\xi_{k}$ for all $k \geqslant k_{T}$. Since $v$ is a good minimizer $v \in W^{1, \infty}(0, \infty)$. By [9, Lemma 2.1], for every fixed $T>0$ there exists a constant $M_{1}(T)$ such that

$$
\begin{aligned}
I\left((-T, T) ; u_{k}\right) & =I\left(\left(-T+\xi_{k}, T+\xi_{k}\right) ; v\right) \\
& \leqslant U_{2 T}\left(X_{v}\left(-T+\xi_{k}\right), X_{v}\left(T+\xi_{k}\right)\right)+M_{1}(T),
\end{aligned}
$$

for all $k \geqslant k_{T}$. Since $X_{v}$ is bounded and $U_{2 T}$ is continuous, it follows that the sequence $\left\{I\left((-T, T) ; u_{k}\right): k \geqslant k_{T}\right\}$ is bounded. Consequently, by [9, Lemma 2.2], $\left\{u_{k}: k \geqslant k_{T}\right\}$ is bounded in $W^{2, \gamma}(-T, T)$. Hence there exists a subsequence $\left\{u_{k_{j}}\right\}$ and a function $u \in W_{l o c}^{2, \gamma}\left(R^{1}\right)$ such that $(2.3)$ (a) holds. Therefore $\left\{\left(u_{k_{j}}, u_{k_{j}}^{\prime}\right)\right\}$ converges uniformly to $\left(u, u^{\prime}\right)$ in $[-T, T]$, for every $T>0$. This implies (2.3)(b).

By [9, Lemma 2.4], for every $T>0$,

$$
\lim _{k \rightarrow \infty} \Gamma\left((-T, T) ; u_{k}\right)=\lim _{k \rightarrow \infty} \Gamma\left(\left(-T+\xi_{k}, T+\xi_{k}\right) ; v\right)=0 .
$$

By Berkovitz [1], $I((-T, T)$; $)$ is weakly lower semicontinuous in $W^{2, \gamma}(-T, T)$. These facts and the continuity of $\pi(\cdot)$ imply that $\Gamma((-T, T) ; u)=0$, for every $T>0$. Thus (2.3)(c) holds.

Finally, if $\left\{X_{v}\left(\tau+\xi_{k}\right)\right\}$ converges, say to $z$, then $X_{u}(\tau)=z$. Suppose that there are two subsequences of $\left\{u_{k}\right\}$ which converge to $u$ and $\tilde{u}$ locally as in (2.3)(a). Then $u$ and $\tilde{u}$ are perfect functions and $X_{u}(\tau)=X_{\tilde{u}}(\tau)=z$. By Proposition $2.2 u \equiv \tilde{u}$.

COROLLARY 2.1. - Let $v \in W_{\text {loc }}^{2,1}[0, \infty)$ be a good function. Then, for every $z \in \Omega(v)$, there exists $u \in W_{\text {loc }}^{2,1}\left(R^{1}\right)$ such that,

$$
\begin{aligned}
& \text { (a) }\left\{\left(u, u^{\prime}\right)(t): t \in R^{1}\right\} \subset \Omega(v), \quad\left(u, u^{\prime}\right)(0)=z, \\
& \text { (b) } \Gamma\left(\left(T_{1}, T_{2}\right) ; u\right)=0, \quad \forall T_{1}, T_{2} \in R^{1} .
\end{aligned}
$$

Proof. - Given $z \in \Omega(v)$ let $\left\{s_{k}\right\}$ be a sequence of positive numbers tending to $\infty$ such that $\left(v, v^{\prime}\right)\left(s_{k}\right) \rightarrow z$. We apply Proposition 2.3 with $\xi_{k}=s_{k}$. Then $\left(u_{k}, u_{k}^{\prime}\right)(0)=$ $\left(v, v^{\prime}\right)\left(s_{k}\right) \rightarrow z$. Consequently the function $u$ mentioned in Proposition 2.3 satisfies (2.5).

Proposition 2.4. - Let $v \in W_{\text {loc }}^{2,1}[0, \infty)$ be a good function. Suppose that $w$ is a periodic minimizer of $\left(P_{\infty}\right)$ such that $\Omega(v) \subset \Omega(w)$. Then $\Omega(v)=\Omega(w)$ and the following assertion holds:

Let $T>0$ be a period of $w$. Then, for every $\varepsilon>0$ there exists $\tau(\varepsilon)>0$ such that for every $\tau \geqslant \tau(\varepsilon)$ there exists $s \in[0, T)$ such that,

$$
\left|\left(v, v^{\prime}\right)(t+\tau)-\left(w, w^{\prime}\right)(s+t)\right| \leqslant \varepsilon, \quad t \in[0, T] .
$$


Proof. - Let $z \in \Omega(v)$ and let $u \in W_{l o c}^{2,1}\left(R^{1}\right)$ be as in Corollary 2.1. Since $X_{u}(0)=z \in$ $\Omega(w)$, there exists $s_{0} \in R^{1}$ such that $X_{u}(0)=X_{w}\left(s_{0}\right)$. Hence, by (2.5)(b) and Proposition 2.2, $u(t)=w\left(t+s_{0}\right)$ for all $t \in R^{1}$. Thus $\Omega(u)=\Omega(w)$ and consequently, by (2.5)(a), $\Omega(w) \subset \Omega(v)$. Since by assumption, $\Omega(v) \subset \Omega(w)$ we conclude that,

$$
\Omega(v)=\Omega(w) .
$$

We turn now to the proof of the second assertion of the proposition. Suppose that it is not valid. Then there exists $\varepsilon>0$ and a strictly increasing sequence of numbers $\left\{T_{k}\right\}_{k=1}^{\infty}$ tending to infinity such that, for every integer $k \geqslant 1$ and every $s \in[0, T)$,

$$
\sup \left\{\left|X_{v}\left(T_{k}+t\right)-X_{w}(s+t)\right|: t \in[0, T]\right\}>\varepsilon .
$$

Apply Proposition 2.3 with $\xi_{k}=T_{k}$. Let $\left\{u_{k_{j}}\right\}$ and $u$ be as in that proposition. Then $u_{k_{j}} \rightarrow u$ in $C^{1}[0, T]$. Therefore, by (2.7),

$$
\sup \left\{\left|X_{u}(t)-X_{w}(s+t)\right|: t \in[0, T]\right\} \geqslant \varepsilon
$$

for every $s \in[0, T)$.

By $(2.3)(\mathrm{b}),\left(u, u^{\prime}\right)(0) \in \Omega(v)=\Omega(w)$. Therefore there exists $s_{0} \in R^{1}$ such that $\left(u, u^{\prime}\right)(0)=\left(w, w^{\prime}\right)\left(s_{0}\right)$. Hence, by $(2.3)(\mathrm{c})$ and Proposition 2.2,

$$
u(t)=w\left(s_{0}+t\right), \quad \forall t \in R^{1} .
$$

Since this contradicts (2.8), the second assertion is established.

\section{Some properties of $c$-optimal minimizers}

LEMMA 3.1. - Let $v \in W_{l o c}^{1,2}[0, \infty)$ be a good function. If $v$ has a limit at infinity, say $\lim _{t \rightarrow \infty} v(t)=d_{0}$, then

$$
\lim _{t \rightarrow \infty} X_{v}(t)=\left(d_{0}, 0\right) \quad \text { and } \quad f\left(d_{0}, 0,0\right)=\mu(f) .
$$

Proof. - Every good function is bounded. Therefore $d_{0}<\infty$. If $z=\left(d_{0}, z_{2}\right) \in \Omega(v)$, then by Corollary 2.1, there exists $u \in W_{l o c}^{2,1}\left(R^{1}\right)$ such that (2.5)(a) holds. Evidently $u \equiv d_{0}$, i.e., $u$ is the constant function with value $d_{0}$. Thus $z=\left(d_{0}, 0\right)$ and, since $z$ was an arbitrary element of $\Omega(v)$, we conclude that $\Omega(v)=\left\{\left(d_{0}, 0\right)\right\}$. Finally, since $u \equiv d_{0}$, (2.5)(b) implies that $\mu(f)=f\left(d_{0}, 0,0\right)$.

Let $h$ be a real function defined in a domain $D \subset R^{1}$. We say that $h$ changes sign in $D$ if there are points $s_{1}, s_{2} \in D$ such that $h\left(s_{1}\right)>0$ and $h\left(s_{2}\right)<0$. If $s_{0} \in D, h\left(s_{0}\right)=0$ and there exists a neighborhood $U$ of $s_{0}$ such that $h\left(s_{1}\right) h\left(s_{2}\right)<0$ whenever $s_{1}, s_{2} \in U$ and $s_{1}<s_{0}<s_{2}$, we say that $s_{0}$ is a turning point of $h$.

LEMmA 3.2. - Suppose that $v$ is a c-optimal minimizer of $\left(P_{\infty}\right)$ such that $v^{\prime}$ changes sign in every neighborhood of $\infty$. Then there exists a strictly increasing sequence of positive numbers $\left\{t_{k}\right\}_{k=1}^{\infty}$ such that $t_{k} \rightarrow \infty$ and, for every integer $k \geqslant 1$, 
(a) $v^{\prime}$ does not change sign in $\left[t_{k}, t_{k+1}\right]$;

(b) $t_{k}$ is a turning point of $v^{\prime}$.

Proof. - Put

$$
E_{j}=\left\{\tau \in(0, \infty): v^{(j)}(\tau)=0\right\}, \quad j=1, \ldots, 4 .
$$

Our assumption implies that $E_{1}$ is unbounded. We claim that $E_{1}$ has no limit points in $(0, \infty)$. Indeed if $t^{*} \in(0, \infty)$ is a limit point of $E_{1}$, then it is also a limit point of $E_{j}, j=1, \ldots, 4$. Consequently $v^{(j)}\left(t^{*}\right)=0, j=1, \ldots, 4$. Since $v$ satisfies the EulerLagrange equation (EL) it follows that $\frac{\partial f}{\partial x_{1}}\left(v\left(t^{*}\right), 0,0\right)=0$. Hence the constant function with value $v\left(t^{*}\right)$ is a solution of (EL). This implies that $v \equiv v\left(t^{*}\right)$, which contradicts our assumption.

Put

$$
E=\left\{T>1: v^{\prime} \text { changes sign in every neighborhood of } T\right\} .
$$

Since $E_{1}$ has no limit points in $(0, \infty)$ it follows that every point in $E$ is a turning point for $v^{\prime}$. We observe that if $v^{\prime}$ changes sign in an interval $D \subset(0, \infty)$, then $D \cap E \neq \emptyset$.

To verify this assertion, pick $s_{1}, s_{2} \in D$ such that $v^{\prime}\left(s_{1}\right) v^{\prime}\left(s_{2}\right)<0$. To fix notation assume that $s_{1}<s_{2}$ and $v^{\prime}\left(s_{1}\right)>0$. If $\tau \in\left(s_{1}, s_{2}\right)$ is a point where $v$ achieves its maximum over $\left[s_{1}, s_{2}\right]$, then $\tau \in E$.

The above assertion and our assumptions imply that $E$ is unbounded. In additon, $E$ has no limit points in $R_{+}$. Therefore $E$ can be ordered so that $E=\left\{t_{k}\right\}_{k=1}^{\infty}$ is a strictly increasing sequence tending to $\infty$. We have already shown that this sequence satisfies (b). Since the intervals $\left(t_{k}, t_{k+1}\right)$ do not intersect $E$ it follows that $v^{\prime}$ does not change sign in any of these intervals.

LEMmA 3.3. - Suppose that $v$ is a c-optimal minimizer of $\left(P_{\infty}\right)$ such that $v^{\prime}$ changes sign in every neighborhood of $\infty$. Let $\left\{t_{k}\right\}_{k=1}^{\infty}$ be a sequence as in Lemma 3.2 and suppose that

$$
\sup \left\{t_{k+1}-t_{k}: k=1,2, \ldots\right\}=\infty .
$$

Then there exists $d_{0} \in R^{1}$ such that $\mu(f)=f\left(d_{0}, 0,0\right)$ and $\left(d_{0}, 0\right) \in \Omega(v)$.

Proof. - Let $\left\{t_{k^{\prime}}\right\}_{k=1}^{\infty}$ be a subsequence such that $t_{k^{\prime}+1}-t_{k^{\prime}} \rightarrow \infty$. Without loss of generality we may assume that $\operatorname{sign} v^{\prime}$ is constant in the set $\bigcup_{k=1}^{\infty}\left(t_{k^{\prime}}, t_{k^{\prime}+1}\right)$. Now apply Proposition 2.3 to $v$ with $\xi_{k}=\left(t_{k^{\prime}+1}+t_{k^{\prime}}\right) / 2$. It follows that the function $u$ mentioned in that proposition is monotone on the whole line and, by (2.3), $u$ is a perfect function. Consequently $u$ has a limit at $\infty$ and, by Lemma 3.1, it satisfies (3.1). Since $\Omega(u) \subset$ $\Omega(v)$, the conclusion of the lemma follows.

Lemma 3.4. - Suppose that $v$ is a c-optimal minimizer of $\left(P_{\infty}\right)$ such that $v^{\prime}$ changes sign in every neighborhood of $\infty$. In addition suppose that

$$
X_{v}\left(s_{1}\right) \neq X_{v}\left(s_{2}\right), \quad \forall s_{1}, s_{2} \in[0, \infty), s_{1} \neq s_{2} .
$$

If $\left\{t_{j}\right\}_{j=1}^{\infty}$ is a sequence as in Lemma 3.2, then: 
(a) If $j \neq k$ then $v\left(t_{j}\right) \neq v\left(t_{k}\right)$.

(b) The sequences $\left\{v\left(t_{2 j}\right)\right\}$ and $\left\{v\left(t_{2 j+1}\right)\right\}$ are eventually monotone. Moreover, if one of them is eventually increasing the other one is eventually decreasing. Let

$$
\lim _{j \rightarrow \infty} v\left(t_{2 j}\right)=d_{0}, \quad \lim _{j \rightarrow \infty} v\left(t_{2 j+1}\right)=d_{1} .
$$

Then, either

(i) $d_{0}=d_{1}$ and (3.1) holds, or

(ii) $d_{0} \neq d_{1}$ and

$$
\inf \left\{t_{k+1}-t_{k}: k=1,2, \ldots\right\}>0 .
$$

Proof. - Put $S_{j}=\left[t_{j}, t_{j+1}\right], j=1,2, \ldots$. Without loss of generality we may assume that $v^{\prime} \geqslant 0$ in $S_{1}$. Then, by Lemma $3.2,(-1)^{j} v^{\prime} \leqslant 0$ in $S_{j}$, for every integer $j \geqslant 1$. Furthermore, in each of these intervals, $v^{\prime}$ vanishes at most at a finite number of points. (Recall that $E_{1}$, the set of zeros of $v^{\prime}$, has no limit points in $(0, \infty)$.) Therefore $v$ is strictly monotone in every interval $S_{j}$. Since $v$ is continuous it follows that $v_{j}:=\left.v\right|_{S_{j}}$ has an inverse $v_{j}^{-1} \in C\left(S_{j}^{*}\right)$, where $S_{j}^{*}=\left[v\left(t_{j}\right), v\left(t_{j+1}\right)\right]$ if $j$ is odd and $S_{j}^{*}=\left[v\left(t_{j+1}\right), v\left(t_{j}\right)\right]$ if $j$ is even. Assumption (3.3) implies that $v\left(t_{j}\right) \neq v\left(t_{i}\right)$ whenever $i \neq j$. Therefore, for every integer $j \geqslant 1$, either $S_{j}^{*} \varsubsetneqq S_{j+1}^{*}$, or $S_{j}^{*} \supsetneqq S_{j+1}^{*}$.

Next we prove the following assertion.

Suppose that, for some integer $j^{\prime} \geqslant 1, S_{j^{\prime}}^{*} \supset S_{j^{\prime}+1}^{*}$. Then

$$
S_{j^{\prime}+i}^{*} \supset S_{j^{\prime}+i+1}^{*}, \quad i=0,1,2, \ldots
$$

To fix ideas, assume that $j^{\prime}$ is odd. (The proof is similar if $j^{\prime}$ is even.) Suppose that (3.6) is not valid for $i=1$. Then

$$
v\left(t_{j^{\prime}}\right)<v\left(t_{j^{\prime}+2}\right)<v\left(t_{j^{\prime}+1}\right)<v\left(t_{j^{\prime}+3}\right) .
$$

Put $h_{j}=v_{j}^{\prime} \circ v_{j}^{-1}$. Then $h_{j} \in C\left(S_{j}^{*}\right)$ and $v^{\prime}(t)=h_{j}(v(t))$ for every $t \in S_{j}$. Furthermore, $h_{k}$ vanishes at the end points of $S_{k}^{*}$ and $(-1)^{k+1} h_{k} \geqslant 0$, for every integer $k \geqslant 1$. Therefore (3.7) implies that $h_{j^{\prime}}$ and $h_{j^{\prime}+2}$ must intersect at some point in $S_{j^{\prime}}^{*} \cap S_{j^{\prime}+2}^{*}=$ $\left[v\left(t_{j^{\prime}+2}\right), v\left(t_{j^{\prime}+1}\right)\right]$. Hence there exist points $s_{1} \in S_{j^{\prime}}$ and $s_{2} \in S_{j^{\prime}+2}$ such that $\left(v, v^{\prime}\right)\left(s_{1}\right)=$ $\left(v, v^{\prime}\right)\left(s_{2}\right)$, which contradicts (3.3). Thus

$$
S_{j^{\prime}}^{*} \supset S_{j^{\prime}+1}^{*} \Rightarrow S_{j^{\prime}+1}^{*} \supset S_{j^{\prime}+2}^{*},
$$

and (3.6) follows.

In view of the above assertion we conclude that, either there exists $j^{\prime} \geqslant 1$ such that (3.6) holds, or

$$
S_{j}^{*} \subset S_{j+1}^{*}, \quad j=1,2, \ldots
$$

If (3.6) holds, then $\left\{v\left(t_{2 j+1}\right)\right\}$ is increasing for $j \geqslant\left(j^{\prime}-1\right) / 2$ and $\left\{v\left(t_{2 j}\right)\right\}$ is decreasing for $j \geqslant\left(j^{\prime}+1\right) / 2$. If (3.8) holds, then $\left\{v\left(t_{2 j+1}\right)\right\}_{j=0}^{\infty}$ is decreasing and $\left\{v\left(t_{2 j}\right)\right\}_{j=1}^{\infty}$ is increasing. 
Since $v$ is bounded it follows that the limits in (3.4) exist. Clearly, if $d_{0}=d_{1}$ then $\lim _{t \rightarrow \infty} v(t)=d_{0}$. Therefore, by Lemma 3.1, statement (3.1) holds. If $d_{0} \neq d_{1},(3.5)$ is a consequence of the boundedness of $v^{\prime}$.

LEMMA 3.5. - Assume that $v$ is a c-optimal minimizer which satisfies (3.3).

(a) If $\mu(f)<\inf _{R^{1}} f(d, 0,0)$, then there exists a periodic minimizer $w$ of $\left(P_{\infty}\right)$ such that $\Omega(w)=\Omega(v)$. Therefore the conclusion of Proposition 2.4 applies to $v$.

(b) If $\mu(f)=\inf _{R^{1}} f(d, 0,0)$, then either there exists a periodic minimizer $w$ as in (a), or there exists $d_{0} \in R^{1}$ such that $\mu(f)=f\left(d_{0}, 0,0\right)$ and $\left(d_{0}, 0\right) \in \Omega(v)$.

Proof. - If $v$ has a limit at infinity, then, by Lemma 3.1, $\mu(f)=\inf _{R^{1}} f(d, 0,0)$ and (3.1) holds. Therefore, in this case, assertion (b) holds. We turn now to the case where $v$ does not have a limit at infinity, making no assumption on the relation between $\mu(f)$ and $\inf _{R^{1}} f(d, 0,0)$. In this case $v^{\prime}$ changes sign in every neighborhood of infinity. Let $\left\{t_{k}\right\}_{k=1}^{\infty}$ be as in Lemma 3.2. If

$$
\sup \left\{t_{k+1}-t_{k}: k=1,2, \ldots\right\}=\infty,
$$

then Lemma 3.3 implies that there exists $d_{0}$ as in (b). Therefore, in order to complete the proof of the lemma it is sufficient to establish the following:

ASSERTION 3.5.1. - If $v$ has no limit at infinity and

$$
\sup \left\{t_{k+1}-t_{k}: k=1,2, \ldots\right\}<\infty,
$$

then there exists a periodic minimizer $w$ such that $\Omega(w)=\Omega(v)$.

Since $v$ has no limit at infinity, it follows that statement (ii) of Lemma 3.4 holds. Without loss of generality, we may assume that $v^{\prime} \geqslant 0$ in $\left(t_{1}, t_{2}\right)$ and consequently $d_{0}>d_{1}$.

Now apply Proposition 2.3 with $\xi_{k}=t_{k}$. Let $u_{k}$ be defined as in that proposition and let $\left\{u_{k_{j}}\right\}$ be a subsequence of $\left\{u_{2 k}\right\}$ such that $\left\{t_{k_{j}+2}-t_{k_{j}}\right\}_{j=1}^{\infty}$ and $\left\{t_{k_{j}+1}-t_{k_{j}}\right\}_{j=1}^{\infty}$ converge and such that (2.3) holds. Clearly $v^{\prime} \leqslant 0$ in $\bigcup_{j=1}^{\infty}\left(t_{2 k}, t_{2 k+1}\right)$ and

$$
X_{u_{2 k}}(0) \rightarrow\left(d_{0}, 0\right), \quad X_{u_{2 k}}\left(t_{2 k+1}-t_{2 k}\right) \rightarrow\left(d_{1}, 0\right) .
$$

Put $u=\lim _{j \rightarrow \infty} u_{k_{j}}, \tau=\lim _{j \rightarrow \infty}\left(t_{k_{j}+2}-t_{k_{j}}\right)$ and $\tau^{\prime}=\lim _{j \rightarrow \infty}\left(t_{k_{j}+1}-t_{k_{j}}\right)$. Note that, by (3.5), $\tau^{\prime}>0$ and, by (3.4) and (3.11),

$$
X_{u}(0)=X_{u}(\tau)=\left(d_{0}, 0\right), \quad X_{u}\left(\tau^{\prime}\right)=\left(d_{1}, 0\right) .
$$

By (2.3) $u$ is perfect and therefore, by Proposition 2.2 and (3.12), $u$ is periodic with period $\tau$. In view of (3.11), the last assertion of Proposition 2.3 implies that the whole sequence $\left\{u_{2 k}\right\}$ converges to $u$ locally as in (2.3)(a). In particular it follows that $u_{2 k} \rightarrow u$ in $C^{1}[-T, T]$ for every $T>0$.

The definition of $u$ and the fact that it is periodic imply that $\Omega(u) \subset \Omega(v)$. Suppose that $\zeta \in \Omega(v)$ and let $\left\{\tau_{j}\right\}$ be a sequence tending to infinity such that $X_{v}\left(\tau_{j}\right) \rightarrow \zeta$. 
Then for every $j$ there exists $k_{j}$ such that $\tau_{j} \in\left[t_{2 k_{j}}, t_{2 k_{j}+2}\right]$. Put $\xi_{j}=\tau_{j}-t_{2 k_{j}}$. Taking a subsequence if necessary, we may assume that $\left\{\xi_{j}\right\}$ converges, say to $\xi$. Hence $X_{v}\left(\tau_{j}\right)=X_{u_{2 k_{j}}}\left(\xi_{j}\right) \rightarrow X_{u}(\xi)$ and consequently $\zeta \in \Omega(u)$. Thus $\Omega(u)=\Omega(v)$ and the assertion is proved.

The following is a consequence of Lemma 3.5 and Corollary 2.1.

Lemma 3.6. - Assume that $v$ is a c-optimal minimizer of $\left(P_{\infty}\right)$. Then there exists a periodic minimizer $w$ such that $\Omega(w) \subset \Omega(v)$.

Proof. - By Corollary 2.1 there exists a perfect function $u$ such that $\Omega(u) \subset \Omega(v)$. If $u$ is periodic, the proof is finished. If $u$ is not periodic, then, by Proposition 2.2, $u$ satisfies (3.3). Therefore, by Lemma 3.5, there exists a periodic minimizer $w$ such that $\Omega(w) \subset \Omega(u)$. In this connection we observe that, if $\mu(f)=f\left(d_{0}, 0,0\right)$, then the constant function with value $d_{0}$ is a minimizer and it is trivially periodic.

Finally we obtain the following uniform boundedness result.

LEMMA 3.7. - The set of c-optimal functions on $R$ is bounded in $W^{1, \infty}(R)$.

Proof. - By [9, Proposition 2.3] the family of periodic minimizers $\mathcal{P}^{f}$ is bounded in $W^{1, \infty}(R)$. Let $M_{f}$ be such a bound.

If $u$ is a function defined on $R$ let $\tilde{u}$ be the function given by $\tilde{u}(t)=u(-t)$. If $g$ is a function defined on $R^{3}$ let $\tilde{g}$ be the function given by $\tilde{g}\left(x_{1}, x_{2}, x_{3}\right)=g\left(x_{1},-x_{2}, x_{3}\right)$. Note that if $f \in \mathfrak{M}$ then $\tilde{f} \in \mathfrak{M}$. It is easy to see that if $u$ is $c$-optimal on $R$, relative to $f$, then $\tilde{u}$ is $c$-optimal on $R$, relative to $\tilde{f}$. Similarly, if $h$ is a periodic minimizer relative to $f$, then $\tilde{h}$ is a periodic minimizer relative to $\tilde{f}$. In particular $\mu(f)=\mu(\tilde{f})$. Consequently, if $u \in \mathcal{T}^{f}(R)$, then, by Lemma 3.6, there exist periodic minimizers $h_{1}, h_{2} \in \mathcal{P}^{f}$ such that

$$
\Omega\left(h_{1}\right) \subset \Omega(u), \quad \Omega\left(\tilde{h}_{2}\right) \subset \Omega(\tilde{u}) .
$$

(If $\mu(f)=\inf _{R} f(t, 0,0)$, then $h_{i}, i=1,2$ may be a constant.) Recall that $\Omega(u)$ denotes the set of limit points of $\left(u, u^{\prime}\right)$ at $+\infty$. Let $\Omega^{\prime}(u)$ denote the set of limit points of $\left(u, u^{\prime}\right)$ at $-\infty$. Clearly, the fact that $\Omega\left(\tilde{h}_{2}\right) \subset \Omega(\tilde{u})$ implies that $\Omega^{\prime}\left(h_{2}\right) \subset$ $\Omega^{\prime}(u)$. Therefore, by (3.26), there exists sequences $\left\{t_{n}\right\}$ and $\left\{s_{n}\right\}$ tending to $+\infty$ such that $\limsup _{n \rightarrow \infty}\left|X_{u}\right|\left(t_{n}\right)<2 M_{f}$ and $\limsup _{n \rightarrow \infty}\left|X_{u}\right|\left(-s_{n}\right)<2 M_{f}$. Now a standard argument as in [10, Lemma 3.1] implies that that there exists a constant $M_{f}^{\prime}$, depending only on $f$, such that, $\|u\|_{W^{1, \infty}(R)} \leqslant M_{f}^{\prime}$.

\section{Proof of Theorems 1.1, 1.2}

One of the main ingredients in our proof is provided by the following result established in [9, Lemma B.5], which is an extension of [14, Lemma 3.7].

Proposition 4.1. - Let $w$ be a periodic minimizer of $\left(P_{\infty}\right)$ and let $\varepsilon>0$. Then there exist numbers $\delta, q>0$ such that the following assertion holds. If

$$
x, y \in R^{2}, \quad d(x, \Omega(w)) \leqslant \delta, \quad d(y, \Omega(w)) \leqslant \delta,
$$


then, for every $\tau \geqslant q$, there exists $v \in W^{2,1}(0, \tau)$ such that

$$
X_{v}(0)=x, \quad X_{v}(\tau)=y, \quad \Gamma((0, \tau) ; v) \leqslant \varepsilon .
$$

The next proposition describes an interesting property of $c$-optimal minimizers. This is another key ingredient in our proof of the main results.

PROPOSITION 4.2. - Assume that $v$ is a c-optimal minimizer of $\left(P_{\infty}\right)$. Then, for every $T, S>0$,

$$
U_{T}\left(X_{v}(0), X_{v}(T)\right)-T \mu(f) \leqslant U_{S}\left(X_{v}(0), X_{v}(T)\right)-S \mu(f) .
$$

Proof. - We prove the result by negation. Suppose that (4.1) does not hold. Then there exist positive numbers $T_{0}, S_{0}$ such that

$$
\left(U_{T_{0}}\left(X_{v}(0), X_{v}\left(T_{0}\right)\right)-T_{0} \mu(f)\right)-\left(U_{S_{0}}\left(X_{v}(0), X_{v}\left(T_{0}\right)\right)-S_{0} \mu(f)\right):=\lambda>0 .
$$

Using (4.2) we show that, for some $T>0$, there exists a function $\bar{v} \in W^{2, \gamma}(0, T)$ such that

$$
X_{\bar{v}}(0)=X_{v}(0), \quad X_{\bar{v}}(T)=X_{v}(T), \quad I((0, T) ; \bar{v})<I((0, T) ; v) .
$$

Obviously this contradicts the assumption that $v$ is $c$-optimal .

By Lemma 3.6 there exists a periodic minimizer $w$ such that $\Omega(w) \subset \Omega(v)$. Let $\delta, q$ be as in Proposition 4.1 with $\varepsilon=\lambda / 4$. Clearly there exist numbers $\tau_{1}, \tau_{2}$ such that

$$
\tau_{1}>T_{0}, \quad \tau_{2}>\tau_{1}+S_{0}+q, \quad d\left(X_{v}\left(\tau_{i}\right), \Omega(w)\right) \leqslant \delta, \quad i=1,2 .
$$

Put $\tau_{1}^{\prime}=\tau_{1}-T_{0}+S_{0}$ so that $\tau_{2}-\tau_{1}^{\prime}>q$. By Proposition 4.1 there exists $h \in W^{2, \gamma}\left(\tau_{1}^{\prime}, \tau_{2}\right)$ such that

$$
X_{h}\left(\tau_{1}^{\prime}\right)=X_{v}\left(\tau_{1}\right), \quad X_{h}\left(\tau_{2}\right)=X_{v}\left(\tau_{2}\right), \quad \Gamma\left(\left(\tau_{1}^{\prime}, \tau_{2}\right) ; h\right) \leqslant \lambda / 4
$$

Put

$$
x=X_{v}(0), \quad y=X_{v}\left(T_{0}\right), \quad D=\left(0, S_{0}\right),
$$

and let $u$ be a minimizer of $\left(P_{D}^{x, y}\right)$. Thus

$$
X_{u}(0)=X_{v}(0), \quad X_{u}\left(S_{0}\right)=X_{v}\left(T_{0}\right), \quad U_{S_{0}}(x, y)=I(D, u) .
$$

Finally define the function $\bar{v}$ in $\left[0, \tau_{2}\right]$ as follows

$$
\bar{v}(t)= \begin{cases}u(t) & t \in\left[0, S_{0}\right], \\ v\left(t-S_{0}+T_{0}\right) & t \in\left(S_{0}, \tau_{1}^{\prime}\right], \\ h(t) & t \in\left(\tau_{1}^{\prime}, \tau_{2}\right] .\end{cases}
$$

From (4.5) and (4.7) it follows that $\bar{v} \in W^{2, \gamma}\left(0, \tau_{2}\right)$ and

$$
X_{\bar{v}}(0)=X_{v}(0), \quad X_{\bar{v}}\left(\tau_{2}\right)=X_{v}\left(\tau_{2}\right) .
$$


Recall that the function $D \mapsto \Gamma(D, v)$ is nonnegative and finitely additive on partitions of bounded intervals (see (1.4)). Therefore, by (4.8), (4.9),

$$
\begin{aligned}
& I\left(\left(0, \tau_{2}\right) ; \bar{v}\right)-I\left(\left(0, \tau_{2}\right) ; v\right) \\
& \quad=\Gamma\left(\left(0, \tau_{2}\right) ; \bar{v}\right)-\Gamma\left(\left(0, \tau_{2}\right) ; v\right) \\
& \quad=\Gamma\left(\left(0, S_{0}\right) ; u\right)+\Gamma\left(\left(T_{0}, \tau_{1}\right) ; v\right)+\Gamma\left(\left(\tau_{1}^{\prime}, \tau_{2}\right) ; h\right)-\Gamma\left(\left(0, \tau_{2}\right) ; v\right) \\
& \quad \leqslant \Gamma\left(\left(0, S_{0}\right) ; u\right)-\Gamma\left(\left(0, T_{0}\right) ; v\right)-\Gamma\left(\left(\tau_{1}, \tau_{2}\right) ; v\right)+\Gamma\left(\left(\tau_{1}^{\prime}, \tau_{2}\right) ; h\right) .
\end{aligned}
$$

Hence, by (4.5) and (4.7),

$$
\begin{aligned}
& I\left(\left(0, \tau_{2}\right) ; \bar{v}\right)-I\left(\left(0, \tau_{2}\right) ; v\right) \\
& \quad \leqslant \Gamma\left(\left(0, S_{0}\right) ; u\right)-\Gamma\left(\left(0, T_{0}\right) ; v\right)+\lambda / 4 \\
& \quad=I\left(\left(0, S_{0}\right) ; u\right)-S_{0} \mu(f)-I\left(\left(0, T_{0}\right) ; v\right)+T_{0} \mu(f)+\lambda / 4 .
\end{aligned}
$$

Since $v$ is $c$-optimal, $I\left(\left(0, T_{0}\right) ; v\right)=U_{T_{0}}(x, y)$ (see (4.6)). Hence, by (4.2), (4.7) and (4.10),

$$
\begin{aligned}
& I\left(\left(0, \tau_{2}\right) ; \bar{v}\right)-I\left(\left(0, \tau_{2}\right) ; v\right) \\
& \quad \leqslant\left(U_{S_{0}}(x, y)-S_{0} \mu(f)\right)-\left(U_{T_{0}}(x, y)-T_{0} \mu(f)\right)+\lambda / 4=-\frac{3}{4} \lambda<0 .
\end{aligned}
$$

Finally this inequality and (4.9) yield (4.3) with $T=\tau_{2}$.

Proof of Theorem 1.1. - Statement (a) is a simple consequence of (b). However we prove it separately and use it as a step in the proof of (b). First we show that if $v$ is a $c$-optimal minimizer satisfying (1.5), then

$$
\Gamma((0, T) ; v)=0 .
$$

By Proposition 4.2,

$$
M:=U_{T+1}\left(X_{v}(0), X_{v}(T+1)\right)-T \mu(f)-U_{1}\left(X_{v}(0), X_{v}(T+1)\right) \leqslant 0 .
$$

On the other hand, since $v$ is a $c$-optimal minimizer,

$$
U_{T+1}\left(X_{v}(0), X_{v}(T+1)\right)=I((0, T+1) ; v) .
$$

Hence, using (1.5) we obtain (with $M$ as in (4.13))

$$
\begin{aligned}
M & =I((0, T+1) ; v)-T \mu(f)-U_{1}\left(X_{v}(T), X_{v}(T+1)\right) \\
& =I((0, T+1) ; v)-T \mu(f)-I((T, T+1) ; v) \\
& =I((0, T) ; v)-T \mu(f)=\Gamma((0, T) ; v) .
\end{aligned}
$$

Since $\Gamma((0, T) ; v) \geqslant 0,(4.13)$ and (4.14) imply (4.12).

Now let $u$ be the periodic function with period $T$ which coincides with $v$ on $[0, T]$. In view of (1.5), $u \in W_{l o c}^{2, \gamma}(R)$ and (4.12) implies that $u$ is a periodic minimizer. Two $c$-optimal minimizers which coincide on an interval are identical because they satisfy the Euler-Lagrange equation (EL). Consequently $v \equiv u$. 
We turn to the proof of (b). Let $v_{1}, v_{2}$ be $c$-optimal minimizers satisfying (1.6), (1.7) for some $t_{1}, t_{2} \in[0, \infty)$ such that $\left(t_{1}, t_{2}\right) \neq(0,0)$. Without loss of generality we assume that $t_{1} \leqslant t_{2}$. First consider the case when $t_{1}=0$. Then $t_{2}>0$ and

$$
X_{v_{1}}(0)=X_{v_{2}}(0)=X_{v_{2}}\left(t_{2}\right)
$$

Consequently, by (a), $v_{2}$ is a periodic minimizer with period $t_{2}$.

Let $u$ be the function given by,

$$
u(t)= \begin{cases}v_{2}(t) & t \in\left[0, t_{2}\right] \\ v_{1}\left(t-t_{2}\right) & t \in\left(t_{2}, \infty\right)\end{cases}
$$

In view of (4.15) it follows that $u \in W^{2, \gamma}(0, T)$ for every $T>0$. We claim that $u$ is $c$-optimal.

Suppose that the claim is false so that there exist arbitrarily large $T$ satisfying the inequality

$$
I((0, T) ; u)>U_{T}\left(X_{u}(0), X_{u}(T)\right) .
$$

Choose $T>t_{2}+1$ such that (4.17) holds. By (4.16) and the fact that $v_{2}$ is a periodic minimizer,

$$
I((0, T) ; u)=I\left(\left(0, t_{2}\right) ; v_{2}\right)+I\left(\left(t_{2}, T\right) ; u\right)=t_{2} \mu(f)+I\left(\left(t_{2}, T\right) ; u\right) .
$$

Since $v_{1}$ is $c$-optimal,

$$
I\left(\left(t_{2}, T\right) ; u\right)=I\left(\left(0, T-t_{2}\right) ; v_{1}\right)=U_{T-t_{2}}\left(X_{v_{1}}(0), X_{v_{1}}\left(T-t_{2}\right)\right) .
$$

By (4.15)-(4.19),

$$
\begin{aligned}
& U_{T}\left(X_{v_{1}}(0), X_{v_{1}}\left(T-t_{2}\right)\right)-T \mu(f) \\
& \quad=U_{T}\left(X_{u}(0), X_{u}(T)\right)-T \mu(f) \\
& \quad<I\left(\left(t_{2}, T\right) ; u\right)-\left(T-t_{2}\right) \mu(f) \\
& \quad=U_{T-t_{2}}\left(X_{v_{1}}(0), X_{v_{1}}\left(T-t_{2}\right)\right)-\left(T-t_{2}\right) \mu(f) .
\end{aligned}
$$

Since this inequality contradicts Proposition 4.2 with respect to $v_{1}$ it follows that $u$ is $c$-optimal.

By (4.16) $u$ coincides with $v_{2}$ in $\left[0, t_{2}\right]$ and with $v_{1}\left(\cdot-t_{2}\right)$ in $\left[t_{2}, \infty\right)$. Since both $v_{1}$ and $v_{2}$ are $c$-optimal we conclude that $u(\cdot) \equiv v_{2}(\cdot)$ and $v_{1}(\cdot) \equiv v_{2}\left(\cdot+t_{2}\right)$. Finally, since $v_{2}$ is periodic with period $t_{2}$ we conclude that $v_{1} \equiv v_{2}$. This proves statement (b) in the case $t_{1}=0$.

Next we consider the case when $0<t_{1} \leqslant t_{2}$. Put

$$
x:=X_{v_{1}}(0)=X_{v_{2}}(0), \quad y:=X_{v_{1}}\left(t_{1}\right)=X_{v_{2}}\left(t_{2}\right) .
$$

By Proposition 4.2 applied to $v_{j}$, with $T=t_{j}$,

$$
U_{t_{j}}(x, y)-t_{j} \mu(f)=\inf \left\{U_{S}(x, y)-S \mu(f): 0<S<\infty\right\}, \quad j=1,2 .
$$


Since $v_{j}$ is $c$-optimal, $U_{t_{j}}(x, y)=I\left(\left(0, t_{j}\right) ; v_{j}\right), j=1,2$. Consequently,

$$
I\left(\left(0, t_{1}\right) ; v_{1}\right)-t_{1} \mu(f)=I\left(\left(0, t_{2}\right) ; v_{2}\right)-t_{2} \mu(f) .
$$

Let $u$ be the function given by,

$$
u(t)= \begin{cases}v_{2}(t) & t \in\left[0, t_{2}\right] \\ v_{1}\left(t-t_{2}+t_{1}\right) & t \in\left(t_{2}, \infty\right) .\end{cases}
$$

In view of (4.21) it follows that $u \in W^{2, \gamma}(0, T)$ for every $T>0$. We claim that $u$ is $c$ optimal. Again the proof is by negation. If the claim is false there exists $T>t_{1}+t_{2}+1$ such that (4.17) holds. Put $T^{*}=T-t_{2}+t_{1}$. Then, using (4.21)-(4.23) and (4.17) we obtain

$$
\begin{aligned}
& U_{T^{*}}\left(X_{v_{1}}(0), X_{v_{1}}\left(T^{*}\right)\right)-T^{*} \mu(f) \\
& \quad=I\left(\left(0, T^{*}\right) ; v_{1}\right)-T^{*} \mu(f) \\
& \quad=I\left(\left(0, t_{1}\right) ; v_{1}\right)-t_{1} \mu(f)+I\left(\left(t_{1}, T^{*}\right) ; v_{1}\right)-\left(T-t_{2}\right) \mu(f) \\
& \quad=I\left(\left(0, t_{2}\right) ; v_{2}\right)-t_{2} \mu(f)+I\left(\left(t_{1}, T^{*}\right) ; v_{1}\right)-\left(T-t_{2}\right) \mu(f) \\
& \quad=I((0, T) ; u)-T \mu(f)>U_{T}\left(X_{u}(0), X_{u}(T)\right)-T \mu(f) \\
& \quad=U_{T}\left(X_{v_{1}}(0), X_{v_{1}}\left(T^{*}\right)\right)-T \mu(f) .
\end{aligned}
$$

Since this inequality contradicts Proposition 4.2 with respect to $v_{1}$ it follows that $u$ is $c$-optimal. As before, the fact that $u, v_{1}, v_{2}$ are $c$-optimal and (4.23) imply that

$$
u(\cdot) \equiv v_{2}(\cdot) \quad \text { and } \quad v_{1}(\cdot) \equiv v_{2}\left(\cdot+t_{2}-t_{1}\right) .
$$

If $t_{1}=t_{2}$ we have $v_{1} \equiv v_{2}$. If $t_{1} \neq t_{2}$, then by (4.21) and (4.25), $X_{v_{2}}(0)=X_{v_{1}}(0)=$ $X_{v_{2}}\left(t_{2}-t_{1}\right)$ and consequently, by part (a), $v_{2}$ is periodic with period $t_{2}-t_{1}$. Hence, by (4.25), $v_{2} \equiv v_{1}$.

Proof of Theorem 1.2. - Suppose that $v$ is a $c$-optimal minimizer. By Theorem 1.1(a), if there exist $s_{1}, s_{2} \in[0, \infty), s_{1} \neq s_{2}$ such that $X_{v}\left(s_{1}\right)=X_{v}\left(s_{2}\right)$, then $v$ is periodic. Therefore it remains to deal with the case where (3.3) holds, namely

$$
X_{v}\left(s_{1}\right) \neq X_{v}\left(s_{2}\right), \quad \forall s_{1}, s_{2} \in[0, \infty), s_{1} \neq s_{2} .
$$

In this case the conclusion of the theorem follows from Lemma 3.5(i).

\section{Perfect minimizers}

An integrand $f \in \mathfrak{M}$ possesses the periodic uniqueness property if problem $\left(P_{\infty}\right)$ has a unique (up to translation) periodic minimizer. It is known that this property holds generically in $\mathfrak{M}$, [9, Theorem 3.1]. When this property holds we can obtain more precise information on the structure of $c$-optimal minimizers. 
THEOREM 5.1. - Suppose that $f \in \mathfrak{M}$ has the periodic uniqueness property. Then every c-optimal minimizer of $\left(P_{\infty}\right)$ is perfect.

Proof. - Recall that, by Proposition 1.2, for every $x \in R^{2}$ there exists an $f$-perfect function $u \in W^{2, \gamma}(0, \infty)$ such that $X_{u}(0)=x$. Furthermore, by Proposition 1.3, every $f$-perfect function on $R_{+}$is a $c$-optimal minimizer.

Let $v$ be a $c$-optimal minimizer of $\left(P_{\infty}\right)$ and let $u$ be a perfect minimizer such that $X_{u}(0)=X_{v}(0)$. By Lemma 3.6, if $w$ is the unique periodic minimizer of $\left(P_{\infty}\right)$, then

$$
\Omega(w) \subset \Omega(u) \cap \Omega(v) .
$$

Suppose that $v$ is not perfect. Then there exists $\tau_{0}>0$ such that

$$
\Gamma\left(\left(0, \tau_{0}\right) ; v\right):=\rho>0 .
$$

Let $\delta$ and $q$ be as in Proposition 4.1 with $\varepsilon=\rho / 2$. By (5.1) there exist arbitrarily large $s_{1}, s_{2}$ such that

$$
d\left(X_{u}\left(s_{1}\right), \Omega(w)\right) \leqslant \delta, \quad d\left(X_{v}\left(s_{2}\right), \Omega(w)\right) \leqslant \delta .
$$

Choose $s_{1}, s_{2}$ satisfying (5.3) such that $s_{1}>\tau_{0}, s_{2}>s_{1}+q$. Then, by Proposition 4.1, there exists $h \in W^{2,1}\left(s_{1}, s_{2}\right)$ such that

$$
X_{h}\left(s_{1}\right)=X_{u}\left(s_{1}\right), \quad X_{h}\left(s_{2}\right)=X_{v}\left(s_{2}\right), \quad \Gamma\left(\left(s_{1}, s_{2}\right) ; h\right) \leqslant \rho / 2 .
$$

Let $\xi$ be the function given by

$$
\xi(t)= \begin{cases}u(t) & t \in\left[0, s_{1}\right), \\ h(t) & t \in\left[s_{1}, s_{2}\right), \\ v(t) & t \in\left[s_{2}, \infty\right)\end{cases}
$$

Then $\xi \in W^{2, \gamma}(0, T)$ for every $T>0$. Recall that $\Gamma$ is a non-negative, additive function on intervals. Consequently, by (5.5), (5.2) and (5.4),

$$
I\left(\left(0, s_{2}\right) ; \xi\right)-I\left(\left(0, s_{2}\right) ; v\right)=\Gamma\left(\left(0, s_{1}\right) ; u\right)+\Gamma\left(\left(s_{1}, s_{2}\right) ; h\right)-\Gamma\left(\left(0, s_{2}\right) ; v\right) \leqslant-\rho / 2 .
$$

Since $X_{\xi}(0)=X_{u}(0)=X_{v}(0)$ and $X_{\xi}\left(s_{2}\right)=X_{v}\left(s_{2}\right)$ this inequality contradicts the fact that $v$ is $c$-optimal .

\section{Proof of Theorem 1.3}

By assumption,

$$
\mu(f)=\inf \left\{f(x, 0,0): x \in R^{1}\right\} .
$$

Let $v \in W_{l o c}^{2,1}[0, \infty) \cap W^{1, \infty}(0, \infty)$ be a $c$-optimal minimizer of $\left(P_{\infty}\right)$ and suppose that $v$ is not periodic. (Otherwise there is nothing to prove.) Then, by Theorem 1.1,

$$
X_{v}\left(s_{1}\right) \neq X_{v}\left(s_{2}\right) \quad \text { for each } s_{1}, s_{2} \text { satisfying } 0 \leqslant s_{1}<s_{2} .
$$


If there exists $d \in R^{1}$ such that $\lim _{t \rightarrow \infty} X_{v}(t)=(d, 0)$, then by Corollary $2.1 \mu(f)=$ $f(d, 0,0)$ and Theorem 1.3 holds. Therefore, in the sequel we assume that

$$
\Omega(v) \text { is not a singleton. }
$$

Consequently Lemmas 3.1 and 3.2 imply that there exists a strictly increasing sequence of positive numbers $\left\{t_{k}\right\}_{k=1}^{\infty}$ such that $t_{k} \rightarrow \infty$ as $k \rightarrow \infty$ and, for every integer $k \geqslant 1$,

(i) $v^{\prime}$ does not change sign in $\left[t_{k}, t_{k+1}\right]$;

(ii) $t_{k}$ is a turning point of $v^{\prime}$.

Without loss of generality we assume that

$$
(-1)^{j} v^{\prime}(t) \leqslant 0 \quad \forall t \in\left[t_{j}, t_{j+1}\right], j=1,2, \ldots
$$

By (6.2) $v$ is strictly monotone in each such interval. By Lemma 3.4 there exists $i_{0} \geqslant 1$ such that either

$$
v\left(t_{2 i+2}\right)>v\left(t_{2 i}\right) \quad \text { and } \quad v\left(t_{2 i+3}\right)<v\left(t_{2 i+1}\right) \quad \forall i \geqslant i_{0},
$$

or

$$
v\left(t_{2 i+2}\right)<v\left(t_{2 i}\right) \text { and } \quad v\left(t_{2 i+3}\right)>v\left(t_{2 i+1}\right) \quad \forall i \geqslant i_{0} .
$$

In either case the following limits exist

$$
z_{2}=\lim _{i \rightarrow \infty} v\left(t_{2 i}\right), \quad z_{1}=\lim _{i \rightarrow \infty} v\left(t_{2 i+1}\right) .
$$

By (A2), $v\left(t_{2 i-1}\right)<v\left(t_{2 i}\right)$, for all $i \geqslant 1$. Hence, $z_{1} \leqslant z_{2}$. By Lemma 3.4, if $z_{2}=z_{1}$, then (3.1) holds with $d_{0}=z_{1}$, which contradicts (A1). Therefore

$$
z_{1}<z_{2}
$$

Consequently, by (6.2) and Lemma 3.4,

$$
\inf \left\{t_{i+1}-t_{i}: i \geqslant 1\right\}>0 .
$$

For every real $s$, let $T_{s}$ denote the translation by amount $s$. Accordingly, since $v$ is defined on $[0, \infty), T_{s} v$ is defined on $[-s, \infty)$ by

$$
T_{s} v(t)=v(s+t), \quad t \geqslant-s .
$$

Put $v_{j}:=T_{t_{j}} v$. By Proposition 2.3, it follows from (6.5) that the sequences $\left\{v_{2 j}\right\}$ and $\left\{v_{2 j+1}\right\}$ converge. More precisely, there exist $u_{l}, u_{r} \in W_{l o c}^{2, \gamma}\left(R^{1}\right)$ such that

$$
v_{2 j+1} \rightarrow u_{l}, v_{2 j} \rightarrow u_{r} \quad \text { as } j \rightarrow \infty \text { weakly in } W^{2, \gamma}(-A, A) \forall A>0
$$

and $u_{l}, u_{r}$ are perfect on $R^{1}$. 
Put

$$
\tau_{j}=t_{j+1}-t_{j}, \quad j=1,2, \ldots
$$

By assumption (A1), $v$ does not posses a limit at infinity. Therefore, if $\left\{\tau_{j}\right\}_{j=1}^{\infty}$ is bounded, Assertion 3.5.1 (see proof of Lemma 3.5), implies that there exists a periodic minimizer $w$ such that $\Omega(v)=\Omega(w)$. Consequently, by Proposition 2.4, (1.8) holds. Therefore, in the sequel we may assume that,

$$
\sup \left\{\tau_{j}\right\}_{j=1}^{\infty}=\infty
$$

In the remaining part of this section we complete the proof of the theorem, under assumptions (A1)-(A3). This part of the proof will be based on several lemmas.

LEMMA 6.1. - If $\left\{\tau_{2 j-1}\right\}_{j=1}^{\infty}$ is bounded, then it converges to a positive number; if it is unbounded, then it tends to infinity. The statement remains valid if $\left\{\tau_{2 j-1}\right\}_{j=1}^{\infty}$ is replaced by $\left\{\tau_{2 j}\right\}_{j=1}^{\infty}$.

Proof. - We prove the assertion for $\left\{\tau_{2 j-1}\right\}_{j=1}^{\infty}$. The proof is similar for $\left\{\tau_{2 j}\right\}_{j=1}^{\infty}$.

Suppose that $\left\{\tau_{2 j-1}\right\}_{j=1}^{\infty}$ is unbounded but it has a finite limit point $\tau$. By (6.7) $\tau>0$. Note that, for every integer $j \geqslant 1, v_{2 j-1}$ is monotone increasing in $\left[0, \tau_{2 j-1}\right)$ and monotone decreasing in $\left[\tau_{2 j-1}, \tau_{2 j-1}+\tau_{2 j}\right]$. Therefore, since $\left\{\tau_{2 j-1}\right\}_{j=1}^{\infty}$ is unbounded, it follows that $u_{l}^{\prime} \geqslant 0$ in $R_{+}$(see (6.9)). Similarly, since $\left\{\tau_{2 j-1}\right\}_{j=1}^{\infty}$ possesses a subsequence which converges to $\tau$, it follows that $u_{l}^{\prime} \leqslant 0$ in $(\tau, \tau+c)$, where $c=\liminf \tau_{2 j}$. Note that by (6.7) $c>0$. These facts imply that $u_{l}^{\prime} \equiv 0$ in $(\tau, \tau+c)$ so that $u_{l}$ has a constant value, say $b$, in this interval. This implies that the constant function with value $b$ is a solution of (EL). Since $u_{l}$ is perfect in $R$, it is also a solution of (EL). Hence $u_{l} \equiv b$. On the other hand, by $(6.5), X_{u_{l}}(0)=\left(z_{1}, 0\right)$ and $X_{u_{l}}(\tau)=\left(z_{2}, 0\right)$. Since, by $(6.6), z_{1}<z_{2}$, we reached a contradiction.

Next suppose that $\left\{\tau_{2 j-1}\right\}_{j=1}^{\infty}$ is bounded but does not converge. Let $\tau$ and $\tau^{\prime}$ be two limit points of the sequence, $\tau<\tau^{\prime}$. By (6.7) $\tau>0$. By the previous argument, $u_{l}^{\prime} \leqslant 0$ in $(\tau, \tau+c)$ and $u_{l}^{\prime} \geqslant 0$ in $\left(0, \tau^{\prime}\right)$. Therefore $u$ is constant in $\left(\tau, c^{\prime}\right)$, where $c^{\prime}=\min \left(\tau^{\prime}, \tau+c\right)$. As before this leads to a contradiction.

Put

$$
\tau_{l}=\lim _{j \rightarrow \infty} \tau_{2 j-1}, \quad \tau_{r}=\lim _{j \rightarrow \infty} \tau_{2 j}
$$

In view of (A3) it remains to deal with the following three cases:

(a) $\tau_{l}=\infty, \tau_{r}<\infty$;

(b) $\tau_{r}=\infty, \tau_{l}<\infty$;

(c) $\tau_{l}=\infty, \tau_{r}=\infty$.

The next lemma describes the behaviour of the limit functions $u_{l}$ and $u_{r}$ (see (6.9)) in each of these cases. We use the following notation. If $u$ is defined in a neighborhood of $+\infty$ or $-\infty$ and possesses a limit there, then

$$
\sigma_{+}(u):=\lim _{t \rightarrow \infty} u(t), \quad \sigma_{-}(u):=\lim _{t \rightarrow-\infty} u(t)
$$

LEMMA 6.2. - (i) If (a) holds, then 


$$
\begin{aligned}
& u_{l}^{\prime}(t) \geqslant 0 \quad \forall t \in\left(-\infty,-\tau_{r}\right), \quad u_{l}^{\prime}(t) \leqslant 0 \quad \forall t \in\left(-\tau_{r}, 0\right), \\
& u_{l}^{\prime}(t) \geqslant 0 \quad \forall t \in(0, \infty), \\
& u_{l}=T_{\tau_{r}} u_{r}, \quad \sigma_{+}\left(u_{l}\right) \leqslant \sigma_{-}\left(u_{r}\right) .
\end{aligned}
$$

(ii) If (b) holds, then

$$
\begin{aligned}
& u_{r}^{\prime}(t) \leqslant 0 \quad \forall t \in\left(-\infty,-\tau_{l}\right), \quad u_{r}^{\prime}(t) \geqslant 0 \quad \forall t \in\left(-\tau_{l}, 0\right), \\
& u_{r}^{\prime}(t) \leqslant 0 \quad \forall t \in(0, \infty), \\
& u_{r}=T_{\tau_{l}} u_{l}, \quad \sigma_{-}\left(u_{l}\right) \leqslant \sigma_{+}\left(u_{r}\right) .
\end{aligned}
$$

(iii) If (c) holds, then

$$
\begin{array}{lc}
u_{l}^{\prime}(t) \geqslant 0, \quad \forall t \geqslant 0, & u_{l}^{\prime}(t) \leqslant 0, \quad \forall t \leqslant 0, \\
u_{r}^{\prime}(t) \geqslant 0, \quad \forall t \leqslant 0, & u_{r}^{\prime}(t) \leqslant 0, \quad \forall t \geqslant 0, \\
\sigma_{+}\left(u_{l}\right) \leqslant \sigma_{-}\left(u_{r}\right), & \sigma_{-}\left(u_{l}\right) \leqslant \sigma_{+}\left(u_{r}\right) .
\end{array}
$$

Proof. - The first part of (i) is a simple consequence of (a) and assumption (A2). Note that $v_{2 j+1}(t)=v_{2 j}\left(t+\tau_{2 j}\right), \forall t \in R^{1}$. Since $\tau_{2 j} \rightarrow \tau_{r}<\infty$ it follows that $u_{l}=T_{\tau_{r}} u_{r}$.

Given $T>0$, choose $j$ sufficiently large so that $2 T<\tau_{2 j-1}$. Then $t_{2 j-1}<T+t_{2 j-1} \leqslant$ $-T+t_{2 j}<t_{2 j}$ and consequently $v_{2 j-1}(T) \leqslant v_{2 j}(-T)$. Letting $j \rightarrow \infty$ we obtain $u_{l}(T) \leqslant u_{r}(-T)$. Taking the limit as $T \rightarrow \infty$ yields $\sigma_{+}\left(u_{l}\right) \leqslant \sigma_{-}\left(u_{r}\right)$. This completes the proof of (i).

Part (ii) is proved exactly in the same way as (i) and part (iii) follows by similar arguments.

Let $S_{j}=\left[t_{j}, t_{j+1}\right], j=1,2, \ldots$ By (A2) and (6.2), $v$ is strictly increasing in $S_{j}$ if $j$ is odd and strictly decreasing if $j$ is even. Hence, the function $t \rightarrow v(t), t \in S_{j}$ is invertible, for every integer $j \geqslant 1$. Its inverse $h_{j}$ is defined on $S_{j}^{*}$ where $S_{j}^{*}=\left[v\left(t_{j}\right), v\left(t_{j+1}\right)\right]$ for odd $j$ and $S_{j}^{*}=\left[v\left(t_{j+1}\right), v\left(t_{j}\right)\right]$ for even $j$.

LEMMA 6.3. - (i) If (6.3) holds, then for every integer $i \geqslant 1$,

$$
\begin{array}{cc}
v^{\prime}\left(h_{2 i-1}(s)\right)<v^{\prime}\left(h_{2 i+1}(s)\right) & \forall s \in S_{2 i-1}^{*}, \\
v^{\prime}\left(h_{2 i}(s)\right)>v^{\prime}\left(h_{2 i+2}(s)\right) & \forall s \in S_{2 i}^{*} .
\end{array}
$$

(ii) If (6.4) holds, then for every integer $i \geqslant 1$,

$$
\begin{gathered}
v^{\prime}\left(h_{2 i+1}(s)\right)<v^{\prime}\left(h_{2 i-1}(s)\right) \quad \forall s \in S_{2 i+1}^{*}, \\
v^{\prime}\left(h_{2 i}(s)\right)<v^{\prime}\left(h_{2 i+2}(s)\right) \quad \forall s \in S_{2 i+2}^{*} .
\end{gathered}
$$

Proof. - We shall prove $\left(6.13^{\prime}\right)$; the other assertions are proved in the same way. Let $i \geqslant 1$ be an integer. We claim that the inequality in $\left(6.13^{\prime}\right)$ holds for $s=v\left(t_{2 i-1}\right)$ and $s=v\left(t_{2 i}\right)$. Indeed $h_{2 i-1}\left(v\left(t_{2 i-1}\right)\right)=t_{2 i-1}$ and, by $(6.3), h_{2 i+1}\left(v\left(t_{2 i-1}\right)\right) \in\left(t_{2 i+1}, t_{2 i+2}\right)$. Similarly $h_{2 i-1}\left(v\left(t_{2 i}\right)\right)=t_{2 i}$ and $h_{2 i+1}\left(v\left(t_{2 i}\right)\right) \in\left(t_{2 i+1}, t_{2 i+2}\right)$. Therefore, if $s=v\left(t_{2 i-1}\right)$ or $s=v\left(t_{2 i}\right)$, then $v^{\prime}\left(h_{2 i-1}(s)\right)=0$ and (by (A2) and (6.2)) $v^{\prime}\left(h_{2 i+1}(s)\right)>0$. 
If $\left(6.13^{\prime}\right)$ does not hold for some $s \in\left(v\left(t_{2 i-1}\right), v\left(t_{2 i}\right)\right)$, then, by the mean value theorem, there exists $\bar{s} \in\left(v\left(t_{2 i-1}\right), v\left(t_{2 i}\right)\right)$ such that $v^{\prime}\left(h_{2 i-1}(\bar{s})\right)=v^{\prime}\left(h_{2 i+1}(\bar{s})\right)$. This implies that $X_{v}\left(h_{2 i-1}(\bar{s})\right)=X_{v}\left(h_{2 i+1}(\bar{s})\right)$, which contradicts $(6.2)$. Thus $\left(6.13^{\prime}\right)$ holds.

At this point it is convenient to introduce the following definition.

DEFINITION 6.1. - Let $V$ be a good function and let $\left\{\xi_{k}\right\}$ be a sequence of positive numbers tending to infinity. Suppose that there exists $t_{0} \in R^{1}$ such that the sequence $\left\{X_{v}\left(t_{0}+\xi_{k}\right)\right\}$ converges. By Proposition 2.3 the sequence of translations $T_{\xi_{k}} V$ converges to a function $u \in W_{\text {loc }}^{2, \gamma}\left(R^{1}\right)$ in the following sense:

$$
T_{\xi_{k}} V \rightarrow u \quad \text { weakly in } W^{2, \gamma}(-T, T), \forall T>0 .
$$

The function $u$ will be called a limiting function of $V$ and the sequence $\left\{\xi_{k}\right\}$ will be called $a$ determining sequence for $u$.

LEMmA 6.4. - Suppose that $V$ is a good function. If $u$ is a limiting function of $V$ then $u$ is perfect and

$$
\Xi(u):=\left\{\left(u(t), u^{\prime}(t)\right): t \in R^{1}\right\} \subset \Omega(V) .
$$

If, in addition, $u$ is monotone in a neighborhood of $+\infty($ resp. $-\infty)$ then $\sigma_{+}(u) \in \mathfrak{D}$ (resp. $\left.\sigma_{-}(u) \in \mathfrak{D}\right)$ and $\lim _{t \rightarrow \infty} u^{\prime}(t)=0\left(\right.$ resp. $\left(\lim _{t \rightarrow-\infty} u^{\prime}(t)=0\right)$.

Proof. - The first statement follows from the proof of Proposition 2.3. If $u$ is monotone near infinity, Lemma 3.1 implies that $\sigma_{+}(u) \in \mathfrak{D}$ and that $\lim _{t \rightarrow \infty} u^{\prime}(t)=0$.

By [9, Lemma 2.2], $\left\{u(\cdot+A): A \in R^{1}\right\}$ is bounded in $W^{2, \gamma}(0,1)$. Therefore, there exists a sequence $\left\{T_{n}\right\}$ tending to $\infty$ such that $\left\{u\left(\cdot-T_{n}\right)\right\}$ converges weakly in $W^{2, \gamma}(0, A)$ for every $A>0$. Put $w_{n}=u\left(\cdot-T_{n}\right)$. If $u$ has a limit at $-\infty$, then $w_{n}(t) \rightarrow \sigma_{-}(u)$ for every real $t$. Therefore the limit of the weak convergence mentioned above is the constant function $w \equiv \sigma_{-}(u)$. By the lower semicontinuity of the functional $I^{f}, I(0, A, w) \leqslant \liminf I\left(0, A, w_{n}\right)$, for every $A>0$. Since $u$ is perfect this implies

$$
\frac{1}{A} I(0, A, w)=f\left(\sigma_{-}(u), 0,0\right) \leqslant \mu(f)+\frac{c}{A},
$$

where $c$ is a constant independent of $A$. Letting $A \rightarrow \infty$ we conclude that $\sigma_{-}(u) \in$ $\mathfrak{D}$.

The next lemma provides the basic block in the construction of $\Omega(v)$. Here $v$ denotes a $c$-optimal minimizer satisfying the assumptions mentioned previously in this section.

LEMMA 6.5. - Let $U_{1}, U_{2}$ be limiting functions of $v$.

(i) Suppose that $\tau_{l}=\lim \tau_{2 j-1}=\infty$. Assume that there exist determining sequences $\left\{s_{i}\right\}$ and $\left\{\xi_{i}\right\}$ for $U_{1}$ and $U_{2}$ respectively, such that

$$
t_{2 i-1} \leqslant s_{i}<\xi_{i} \leqslant t_{2 i} \quad \forall i \geqslant 2, \quad \lim _{i \rightarrow \infty}\left(\xi_{i}-s_{i}\right)=\infty .
$$

Then $U_{1}$ is monotone increasing in $(0, \infty), U_{2}$ is monotone increasing in $(-\infty, 0)$ and

$$
\sigma_{+}\left(U_{1}\right) \leqslant \sigma_{-}\left(U_{2}\right) .
$$


If, in addition,

$$
\sigma_{+}\left(U_{1}\right)<\sigma_{-}\left(U_{2}\right)
$$

then there exists a limiting function of $v$, say $w$, such that $w$ is monotone increasing on the whole line and

$$
\sigma_{+}\left(U_{1}\right) \leqslant \sigma_{-}(w) \leqslant \sigma_{+}(w) \leqslant \sigma_{-}\left(U_{2}\right) .
$$

Furthermore $w$ has a determining sequence $\left\{\theta_{i}\right\}_{i=1}^{\infty}$ such that

$$
s_{i}<\theta_{i}<\xi_{i} \forall i \geqslant 1, \quad \lim \left(\theta_{i}-s_{i}\right)=\lim \left(\xi_{i}-\theta_{i}\right)=\infty .
$$

(ii) Suppose that $\tau_{r}=\lim \tau_{2 j}=\infty$. Assume that there exist determining sequences $\left\{s_{i}\right\}$ and $\left\{\xi_{i}\right\}$ for $U_{1}$ and $U_{2}$ respectively, such that

$$
t_{2 i} \leqslant s_{i}<\xi_{i} \leqslant t_{2 i+1} \forall i \geqslant 1, \quad \lim _{i \rightarrow \infty}\left(\xi_{i}-s_{i}\right)=\infty .
$$

Then $U_{1}$ is monotone decreasing in $(0, \infty), U_{2}$ is monotone decreasing in $(-\infty, 0)$ and

$$
\sigma_{+}\left(U_{1}\right) \geqslant \sigma_{-}\left(U_{2}\right)
$$

If, in addition,

$$
\sigma_{+}\left(U_{1}\right)>\sigma_{-}\left(U_{2}\right)
$$

then there exists a limiting function of $v$, say $w^{*}$, such that $w^{*}$ is monotone decreasing on the whole line and

$$
\sigma_{+}\left(U_{1}\right) \geqslant \sigma_{-}(w) \geqslant \sigma_{+}(w) \geqslant \sigma_{-}\left(U_{2}\right) .
$$

Furthermore $w^{*}$ has a determining sequence $\left\{\theta_{i}\right\}_{i=1}^{\infty}$ such that (6.20) holds.

Proof. - We shall prove part (i). Part (ii) is proved exactly in the same way.

The assertion concerning the monotonicity of $U_{1}$ and $U_{2}$ in $(0, \infty)$ and $(-\infty, 0)$ respectively follows from (6.16) and (A2). Inequality (6.17) can be verified by the same argument as in the proof of the corresponding inequality in Lemma 6.2(i).

Assume that (6.18) holds and put $\rho=\left(\sigma_{-}\left(U_{2}\right)-\sigma_{+}\left(U_{1}\right)\right) / 4$. Since $U_{1}(0) \leqslant$ $\sigma_{+}\left(U_{1}\right), U_{2}(0) \geqslant \sigma_{-}\left(U_{2}\right)$, it follows that, for all sufficiently large $i$,

$$
v\left(s_{i}\right)<\sigma_{+}\left(U_{1}\right)+\rho, \quad v\left(\xi_{i}\right)>\sigma_{-}\left(U_{2}\right)-\rho .
$$

Note that $\sigma_{+}\left(U_{1}\right)+\rho<\left(\sigma_{+}\left(U_{1}\right)+\sigma_{-}\left(U_{2}\right)\right) / 2<\sigma_{-}\left(U_{2}\right)-\rho$. Therefore, by (6.21), for all sufficiently large $i$,

$$
\exists \theta_{i} \in\left(s_{i}, \xi_{i}\right): v\left(\theta_{i}\right)=\left(\sigma_{+}\left(U_{1}\right)+\sigma_{-}\left(U_{2}\right)\right) / 2 .
$$

Hence, by Lemma 6.3, the sequence $\left\{v^{\prime}\left(\theta_{i}\right)\right\}$ is monotone. By Proposition 2.3, $\left\{T_{\theta_{i}} v\right\}$ converges to a limiting function $w$, which is perfect on $R$. By (6.22),

$$
w(0)=\left(\sigma_{+}\left(U_{1}\right)+\sigma_{-}\left(U_{2}\right)\right) / 2 .
$$


In order to verify (6.20), assume that there exists a subsequence $\left\{\theta_{i_{k}}\right\}$ such that $\theta_{i_{k}}-s_{i_{k}} \rightarrow A<\infty$. Then $T_{\theta_{i_{k}}} v \rightarrow T_{A} U_{1}$ so that $w=T_{A} U_{1}$. Hence $w(0)=U_{1}(A) \leqslant$ $\sigma_{+}\left(U_{1}\right)$, which contradicts (6.23). Thus $\lim \left(\theta_{i}-s_{i}\right)=\infty$. Similarly one shows that $\lim \left(\xi_{i}-\theta_{i}\right)=\infty$. Further, (6.20) and (A2) imply that $w$ is monotone increasing on the whole line and that (6.19) holds. This completes the proof.

Up to this point we did not make use of the assumption that $\mathfrak{D}$ is finite. This assumption is needed in the remaining part of the proof.

LEMMA 6.6. - Assume that $\mathfrak{D}$ is finite.

(i) Suppose that $\tau_{l}=\infty$ and that $\sigma_{+}\left(u_{l}\right)<\sigma_{-}\left(u_{r}\right)$. Then $\Omega(v)$ contains a set $E_{1}$ of the form

$$
E_{1}=\bigcup_{i=1}^{k} \bar{\Xi}\left(u_{i}\right),
$$

where $k<\infty$ and $u_{i}, i=1, \ldots, k$, is a limiting function of $v$, which is perfect and monotone increasing on the whole line, such that

$$
\begin{aligned}
& \sigma_{+}\left(u_{l}\right)=\sigma_{-}\left(u_{1}\right), \quad \sigma_{+}\left(u_{k}\right)=\sigma_{-}\left(u_{r}\right), \\
& \sigma_{-}\left(u_{i}\right) \leqslant \sigma_{+}\left(u_{i}\right), \quad i=1, \ldots, k, \quad \sigma_{+}\left(u_{i}\right)=\sigma_{-}\left(u_{i+1}\right), \quad i=1, \ldots, k-1 .
\end{aligned}
$$

Furthermore each limiting function $u_{i}, i=1, \ldots, k$, has a determining sequence $\left\{\theta_{i, j}\right\}_{j=1}^{\infty}$ such that

$$
\begin{aligned}
& t_{2 j-1}=: \theta_{0, j}<\theta_{1, j}<\cdots<\theta_{k, j}<t_{2 j}=: \theta_{k+1, j}, \quad j=1,2, \ldots, \\
& \lim _{j \rightarrow \infty} \theta_{i+1, j}-\theta_{i, j}=\infty, \quad i=0, \ldots, k
\end{aligned}
$$

(ii) Suppose that $\tau_{r}=\infty$ and that $\sigma_{-}\left(u_{l}\right)<\sigma_{+}\left(u_{r}\right)$. Then $\Omega(v)$ contains a set $E_{2}$ of the form

$$
E_{2}=\bigcup_{i=1}^{k^{\prime}} \bar{\Xi}\left(u_{i}^{*}\right),
$$

where $k^{\prime}<\infty$ and $u_{i}^{*}, i=1, \ldots, k^{\prime}$, is a perfect function, monotone decreasing on the whole line, such that

$$
\begin{aligned}
& \sigma_{-}\left(u_{l}\right)=\sigma_{+}\left(u_{1}^{*}\right), \quad \sigma_{-}\left(u_{k}^{*}\right)=\sigma_{+}\left(u_{r}\right), \\
& \sigma_{+}\left(u_{i}^{*}\right) \leqslant \sigma_{-}\left(u_{i}^{*}\right), \quad i=1, \ldots, k^{\prime}, \quad \sigma_{-}\left(u_{i}^{*}\right)=\sigma_{+}\left(u_{i+1}^{*}\right), \quad i=1, \ldots, k^{\prime}-1 .
\end{aligned}
$$


Furthermore each limiting function $u_{i}^{*}, i=1, \ldots, k^{\prime}$, has a determining sequence $\left\{\theta_{i, j}^{*}\right\}_{j=1}^{\infty}$ such that

$$
\begin{aligned}
& t_{2 j}=: \theta_{0, j}^{*}<\theta_{1, j}^{*}<\cdots<\theta_{k^{\prime}, j}^{*}<t_{2 j+1}=: \theta_{k^{\prime}+1, j}^{*}, \quad j=1,2, \ldots, \\
& \lim _{j \rightarrow \infty} \theta_{i+1, j}^{*}-\theta_{i, j}^{*}=\infty, \quad i=0, \ldots, k^{\prime} .
\end{aligned}
$$

Proof. - By Lemma 6.2(i), the assumptions of assertion (i) of the present lemma imply that the conditions of Lemma 6.5(i) are satisfied with respect to $U_{1}=u_{l}$ and $U_{2}=u_{r}$. By repeatedly applying Lemma 6.5 we obtain a sequence of limiting functions $w_{1}, w_{2}, \ldots$ such that each function $w_{i}$ is monotone increasing and

$$
\begin{aligned}
& \left(\sigma_{-}\left(w_{i}\right), \sigma_{+}\left(w_{i}\right)\right) \subset\left(\sigma_{+}\left(u_{l}\right), \sigma_{-}\left(u_{r}\right)\right) \quad \forall i, \\
& \left(\sigma_{-}\left(w_{i}\right), \sigma_{+}\left(w_{i}\right)\right) \cap\left(\sigma_{-}\left(w_{j}\right), \sigma_{+}\left(w_{j}\right)\right)=\emptyset \quad \text { for } i \neq j .
\end{aligned}
$$

By Lemma 6.4,

$$
\sigma_{+}\left(u_{l}\right), \sigma_{-}\left(u_{r}\right), \sigma_{-}\left(w_{i}\right), \sigma_{+}\left(w_{i}\right) \in \mathfrak{D} .
$$

Since $\mathfrak{D}$ is finite, (6.26) and (6.27) imply that the sequence of limiting functions constructed above is finite. This completes the proof of part (i). Part (ii) is proved in the same way.

Completion of proof of Theorem 1.3. - If $\tau_{l}<\infty$ or $\sigma_{+}\left(u_{l}\right)=\sigma_{-}\left(u_{r}\right)$ put $E_{1}=\emptyset$; otherwise let $E_{1}$ be as in Lemma 6.6(i). If $\tau_{r}<\infty$ or $\sigma_{-}\left(u_{l}\right)=\sigma_{+}\left(u_{r}\right)$ put $E_{2}=\emptyset$; otherwise let $E_{2}$ be as in Lemma 6.6(ii). To complete the proof we shall show that

$$
\Omega(v)=\bar{\Xi}\left(u_{l}\right) \cup \bar{\Xi}\left(u_{r}\right) \cup E_{1} \cup E_{2} .
$$

Denote the set on the right hand side of (6.28) by $K$. Obviously $K \subset \Omega(v)$. Therefore it remains to show that

$$
\Omega(v) \subset K .
$$

We partition $\Omega(v)$ into two subsets $\Omega_{l}(v), \Omega_{r}(v)$ defined as follows. Let

$$
\mathfrak{T}_{l}:=\bigcup_{j=1}^{\infty}\left[t_{2 j-1}, t_{2 j}\right], \quad \mathfrak{T}_{r}:=\bigcup_{j=1}^{\infty}\left[t_{2 j}, t_{2 j+1}\right] .
$$

If $\xi \in \Omega(v)$ and $\left\{s_{j}\right\}_{j=1}^{\infty}$ is a sequence of positive numbers such that $s_{j} \rightarrow \infty$ and $X_{v}\left(s_{j}\right) \rightarrow \xi$, we say that $\left\{s_{j}\right\}$ is a determining sequence for $\xi$.

A point $\xi \in \Omega(v)$ belongs to $\Omega_{l}(v)$ if there exists a determining sequence for $\xi$ contained in $\mathfrak{T}_{l}$. Put $\Omega_{r}(v):=\Omega(v) \backslash \Omega_{l}(v)$. Clearly, if $\xi \in \Omega_{r}(v)$ there exists a determining sequence for $\xi$ contained in $\mathfrak{T}_{r}$. However it is possible that a point $\xi \in \Omega(v)$ possesses determining sequences in both $\mathfrak{T}_{l}$ and $\mathfrak{T}_{r}$.

For any $u \in C^{1}\left(R^{1}\right)$ put,

$$
\Xi_{+}(u)=\left\{\left(u(t), u^{\prime}(t)\right): t \geqslant 0\right\}, \quad \Xi_{-}(u)=\left\{\left(u(t), u^{\prime}(t)\right): t \leqslant 0\right\} .
$$


To complete the proof, we shall establish the following assertions.

$$
\begin{aligned}
& \text { I. } \quad \xi \in \Omega_{l}(v), \tau_{l}<\infty \Rightarrow \xi \in \bar{\Xi}\left(u_{l}\right), \\
& \text { II. } \xi \in \Omega_{l}(v), \tau_{l}=\infty \Rightarrow \xi \in E_{1} \cup \bar{\Xi}_{+}\left(u_{l}\right) \cup \bar{\Xi}_{-}\left(u_{r}\right), \\
& \text { III. } \xi \in \Omega_{r}(v), \tau_{r}<\infty \Rightarrow \xi \in \bar{\Xi}\left(u_{r}\right), \\
& \text { IV. } \xi \in \Omega_{r}(v), \tau_{r}=\infty \Rightarrow \xi \in E_{2} \cup \bar{\Xi}_{+}\left(u_{r}\right) \cup \bar{\Xi}_{-}\left(u_{l}\right) .
\end{aligned}
$$

Proof of $(C) I$. - Let $\left\{s_{n}\right\}_{n=1}^{\infty}$ be a determining sequence for $\xi$, contained in $\mathfrak{T}_{l}$. Extracting a subsequence if necessary, we may assume that $s_{n} \in\left[t_{2 j_{n}-1}, t_{2 j_{n}}\right], n=$ $1,2, \ldots$, where $\left\{j_{n}\right\}$ is strictly increasing and $\left\{s_{n}-t_{2 j_{n}-1}\right\}$ converges to a number $A$. Then $\xi=\lim _{n \rightarrow \infty} X_{v}\left(s_{n}\right)=X_{u_{l}}(A)$.

Proof of $(C) I I$. - Let $\left\{s_{n}\right\}_{n=1}^{\infty}$ be a determining sequence for $\xi$, contained in $\mathfrak{T}_{l}$. Using the notation of Lemma 6.6(i), we note that there exists a subsequence of $\left\{s_{n}\right\}$ (still denoted by $\left.\left\{s_{n}\right\}\right)$ and an integer $q, 0 \leqslant q \leqslant k$, such that $s_{n} \in\left[\theta_{q, j_{n}}, \theta_{q+1, j_{n}}\right], n=1,2, \ldots$, with $\left\{j_{n}\right\}$ strictly increasing.

By the same argument as in the first part we obtain,

$$
\begin{aligned}
& \liminf _{n \rightarrow \infty}\left(s_{n}-\theta_{q, j_{n}}\right)<\infty \Rightarrow \xi \in \Xi_{+}\left(u_{q}\right), \\
& \liminf _{n \rightarrow \infty}\left(\theta_{q+1, j_{n}}-s_{n}\right)<\infty \Rightarrow \xi \in \Xi_{-}\left(u_{q+1}\right) .
\end{aligned}
$$

We claim that,

$$
\begin{gathered}
\liminf _{n \rightarrow \infty}\left(s_{n}-\theta_{q, j_{n}}\right)=\liminf _{n \rightarrow \infty}\left(\theta_{q+1, j_{n}}-s_{n}\right)=\infty \\
\Rightarrow \xi=\left(\sigma_{+}\left(u_{q}\right), 0\right)=\left(\sigma_{-}\left(u_{q+1}\right), 0\right) .
\end{gathered}
$$

Let $L>0$. Then, for sufficiently large $n, s_{n}-\theta_{q, j_{n}}>L$ and $\theta_{q+1, j_{n}}-s_{n}>L$. Consequently, using (A2),

$$
\begin{gathered}
v\left(s_{n}\right) \geqslant v\left(L+\theta_{q, j_{n}}\right)=T_{\theta_{q, j_{n}}} v(L) \rightarrow u_{q}(L), \\
v\left(s_{n}\right) \leqslant v\left(\theta_{q+1, j_{n}}-L\right)=T_{\theta_{q+1, j n}} v(-L) \rightarrow u_{q+1}(-L) .
\end{gathered}
$$

Hence, letting first $n \rightarrow \infty$ and secondly $L \rightarrow \infty$, we obtain $\sigma_{+}\left(u_{q}\right) \leqslant \lim _{n \rightarrow \infty} v\left(s_{n}\right) \leqslant$ $\sigma_{-}\left(u_{q+1}\right)$. Since $\sigma_{+}\left(u_{q}\right)=\sigma_{-}\left(u_{q+1}\right)$, it follows that

$$
\lim _{n \rightarrow \infty} v\left(s_{n}\right)=\sigma_{+}\left(u_{q}\right) .
$$

The same argument shows that, for every $\delta>0$,

$$
\lim _{n \rightarrow \infty} v\left(s_{n}+\delta\right)=\sigma_{+}\left(u_{q}\right) .
$$

By (A2), $v^{\prime}\left(s_{n}\right) \geqslant 0$ for all $n$. Suppose that $\lim \sup v^{\prime}\left(s_{n}\right)>0$. Extracting a subsequence if necessary, we may assume that $\lim v^{\prime}\left(s_{n}\right)=\rho>0$. By [9, Lemma 2.2] $v^{\prime}$ is uniformly 
continuous on $[0, \infty)$. Consequently, if $\delta>0$ is sufficiently small, $v\left(s_{n}+\delta\right)-v\left(s_{n}\right)>$ $\delta \rho / 2$ for all sufficiently large $n$. As this contradicts (6.33) we conclude that $\lim v^{\prime}\left(s_{n}\right)=0$ and (6.31) is proved. Clearly (6.30) and (6.31) imply the conclusion of (C)II.

Assertions III and IV of (C) are proved exactly in the same way as I and II. Finally (C) implies (6.28). We note that, by construction, $K$ is compact and connected and that (1.10) follows from Lemma 6.4. This completes the proof of the theorem.

\section{REFERENCES}

[1] L.D. Berkovitz, Lower semicontinuity of integral functionals, Amer. Math. Soc. 192 (1974) 51-57.

[2] B.D. Coleman, Necking and drawing in polymeric fibers under tension, Arch. Rational Mech. Anal. 83 (1983) 115-137.

[3] B.D. Coleman, On the cold drawing of polymers, Comp. \& Math. with Appls. 11, 35-65.

[4] B.D. Coleman, M. Marcus, V.J. Mizel, On the thermodynamics of periodic phases, Arch. Rational Mech. Anal. 117 (1992) 321-347.

[5] A. Leizarowitz, Infinite horizon autonomous systems with unbounded cost, Appl. Math. Optim. 13 (1985) 19-43.

[6] A. Leizarowitz, V.J. Mizel, One dimensional infinite horizon variational problems arising in continuum mechanics, Arch. Rational Mech. Anal. 106 (1989) 161-194.

[7] M. Marcus, Uniform estimates for a variational problem with small parameters, Arch. Rational Mech. Anal. 124 (1993) 67-98.

[8] M. Marcus, Universal properties of stable states of a free energy model with small parameters, Cal. Var. 6 (1998) 123-142.

[9] M. Marcus, A.J. Zaslavski, The structure of extremals of a class of second order variational problems, Ann. Inst. H. Poincare Anal. non Lineare 16 (1999) 593-629.

[10] M. Marcus, A.J. Zaslavski, On a class of second order variational problems with constraints, Israel J. Math. 111 (1999) 1-28.

[11] V.J. Mizel, L.A. Peletier, W.C. Troy, Periodic phases in second order materials, Arch. Rational Mech. Anal. 145 (1998) 343-382.

[12] A.J. Zaslavski, The existence of periodic minimal energy configurations for onedimensional infinite horizon variational problems arising in continuum mechanics, J. Math. Anal. Appl. 194 (1995) 459-476.

[13] A.J. Zaslavski, The existence structure of extremals for a class of second order infinite horizon variational problems, J. Math. Anal. Appl 194 (1995) 660-696.

[14] A.J. Zaslavski, Structure of extremals for one-dimensional variational problems arising in continuum mechanics, J. Math. Anal. Appl. 198 (1996) 893-921. 